RYERSON UNIVERSITY

FACULTY OF ENGINEERING, ARCHITECTURE AND SCIENCE DEPARTMENT OF AEROSPACE ENGINEERING

\title{
DESIGN AND AERODYNAMIC ANALYSIS OF COMPLIANT MECHANISM BASED MORPHING WINGS
}

Stephen D. Sharp

AER870 Aerospace Engineering Thesis - Final Report

Faculty Advisor: Dr. Seyed M. Hashemi

Date: April 2020 


\section{Acknowledgements}

Firstly, the author would like to thank his supervisor, Dr. Seyed Hashemi. His mentorship, patience, and insight were crucial to the success of this project. As the authors first foray into a research project of this scale, Dr. Hashemi's endlessly positive attitude and reassurance of the value of the work kept the author motivated and curious to keep learning more. He provided the author with the freedom to take the project in any direction he wanted, but never strayed from providing the guidance required to keep him on track. The author owes him much of this projects success and is grateful to have shared this experience.

Words can't express how grateful the author is for his family. Without his parents' continuous emotional (and financial) support, none of his academic and professional achievements could have been possible. He would also like to thank his grandfather. His grandfather is his number one supporter, and nothing brings him more joy than the feeling that he is making his grandfather proud. Finally, the author would like to thank his partner Haley. Over the past 5 years, her endless patience, love, and support have allowed the author to push himself to achieve whatever he set my mind to. To his whole family; thank you all, he owes you everything. 


\section{Abstract}

Aircraft today use discrete control surface, typically mounted using pin and sliding joints. These designs can lead to high part-count assemblies and backlash within the assemblies that require lubrication and frequent maintenance. These wing designs also feature fixed dimensions and do not allow for geometry changes mid-flight. These limitations lead to a compromised design that must work relatively well in all situations. This causes inefficiencies in all stages of flight.

The Wright brothers, who achieved the first successful powered flight did not use these techniques. Instead they used a system on cables to apply tension and bend the wings to changes their angle of attack. They called this technique wing warping. As aviation advanced it quickly moved from the wing-warping technique towards the discrete element control surfaces. However, there is renewed interest in techniques such as wing warping as the idea of morphing wings becomes more prevalent in aerospace research. Morphing wings would allow for changing major characteristics, such as camber, span, sweep, etc. of the wing mid-flight and allow for continuous optimization through all stages of its mission.

The design covered in this thesis was centered around camber morphing of the wing in flight. Biomimicry played a large role in the design, with research into the skeletal systems of birds and fish used to dictate the rib structures. This bio-inspired path led to the use of compliant mechanisms for the ribs. This choice allowed for a low part-count and zero-backlash design that would require no maintenance and have a very long service life due to an extremely low amount of fatigue. Several design iterations were tested with different common desktop 3-D printing materials. The final rib design was made of PETG and whose compliant shape was directly inspired by the skeletal structure of the spine of a fish. The design proved to be extremely reliable and robust.

Skin design has long been one of the biggest hurdles of morphing wing design. Most research reviewed in this paper used an elastomer style skin that was pre-stretched to reduce buckling under compression. Through testing it was found that this method is difficult and unreliable to maintain a smooth and continuous surface. Even when pre-stretching, the elastomer would fatigue and buckle under compression. The final design was a PETG panel with a web and flange that would interact with the rib structure and was able to translate chordwise along the rib as the wing altered its camber. The skin had built-in flexures to reduce bending actuation forces. The wing also featured a rigid leading-edge skin panel with which the other skin panels would be able to slide under to maintain skin coverage under both extension and compression of the wing surfaces. This however led to aerodynamic problems that were discovered in the CFD analysis.

The wing was prepared for CFD using finite element analysis to produced morphed wing bodies for a $0,10,20$, and 30-degree trailing edge deflection angles. A model was also produced of the same base airfoil (NACA 0018) with a hinged flap of $30 \%$ chord length deflected by the same amount to serve as a performance benchmark for the morphing wing. The main criteria used to evaluate the performance were the lift, drag, and lift-to-drag ratios. For the $0^{\circ}$ tests, the morphing wing had up to almost $29 \%$ higher drag at high speeds. The results showed that the $10^{\circ}$ deflection tests found up to a $115 \%$ increase in lift over the hinged flap design and a lift-to-drag ratio of up to $161 \%$ higher for the morphing wing. The $20^{\circ}$ and $30^{\circ}$ tests saw the lift advantage of the morphing wing decrease but on average across all tests, the morphing wing had a lift coefficient higher than the hinged flap by $43 \%$. Additionally, for the large deflection tests the hinged flap had up to a $60.5 \%$ advantage in lift-to-drag ratio. 
The computational fluid dynamic analysis showed that due to the larger effective angle of attack and the step-down in the skin of the morphing wing, at larger deflection angles the flow would separate much earlier along the chord. Therefore, based on the analysis, the morphing wing would create a substantial performance and efficiency gains when wing trailing edge deflection was kept below $20^{\circ}$. This meant it would be suitable for stages of flight such as takeoff and climb.

Planned future work aims to reduce the $0^{0}$ drag of the morphing wing as well as the early flow separation at high angles of deflection. It is assumed, that by scaling up the wing, the proportion of the step size will decrease dramatically and as a result would improve the flow characteristics. Additionally, the placement and rotational limits of the flexures can be tested further to optimize the morphed shape to reduce the severity of the adverse pressure gradient along the upper surface when in high deflection states. With continued work on improving the flow separation, this design proves promising for even high-deflection cases. Overall the V4 rib design and the accompanying compliant skin panel design were very successful for their initial tests. 


\section{Table of Contents}

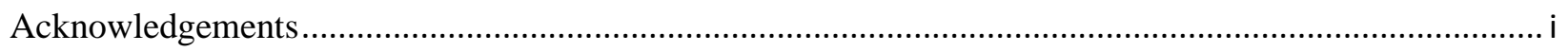

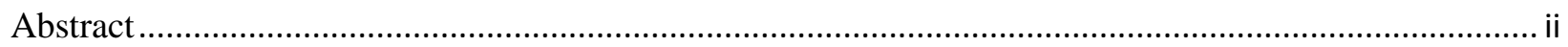

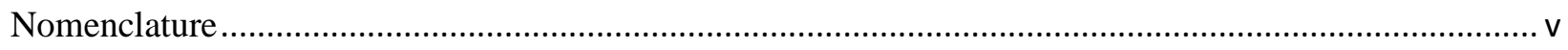

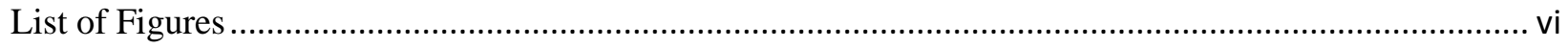

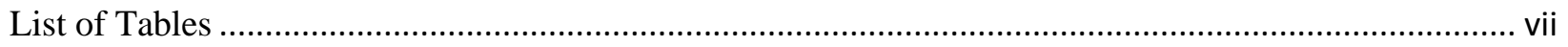

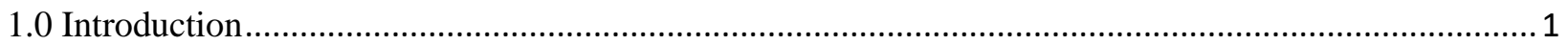

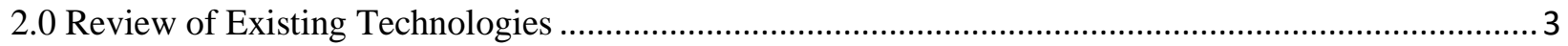

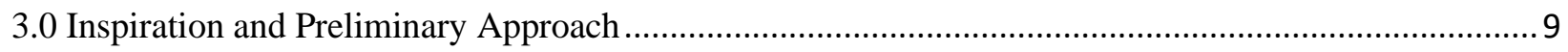

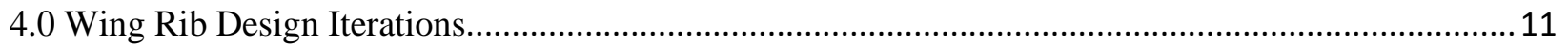

4.1 V1 - 4-Bar Compliant Mechanism ..................................................................................... 11

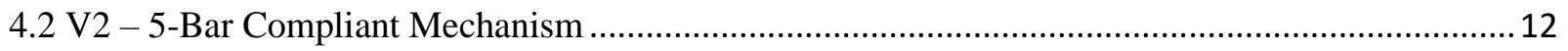

4.3 V3 - Compliant Multi-Segment Spine Using Flexible Material................................................... 12

4.4 V4 - Compliant Multi-Segment Spine with Step-Down Skin ..................................................... 13

4.5 V5 - Compliant Multi-Segment Spine with Split Trailing Edge ................................................ 15

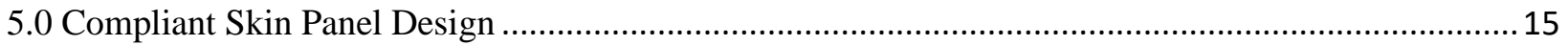

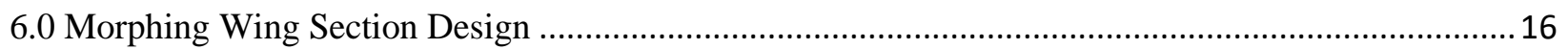

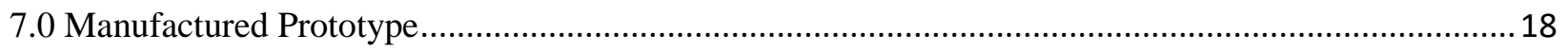

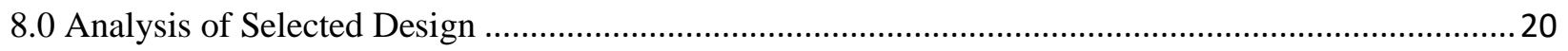

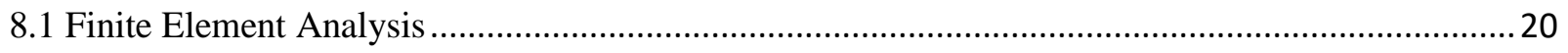

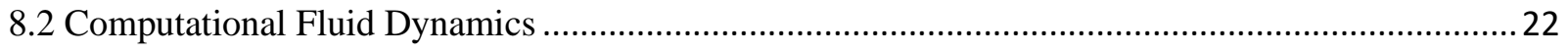

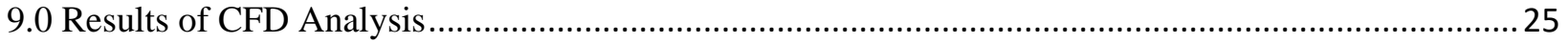

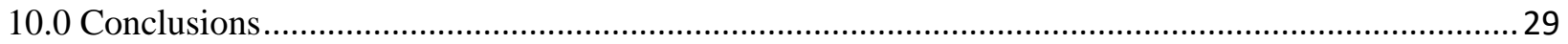

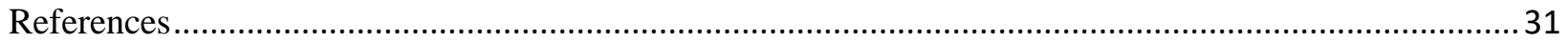

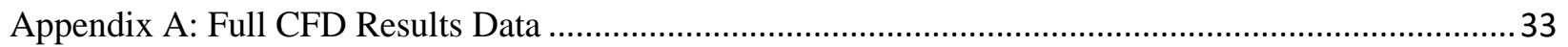

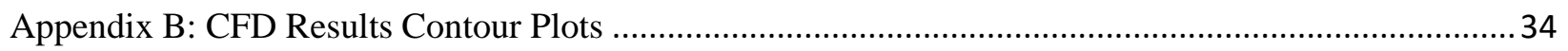

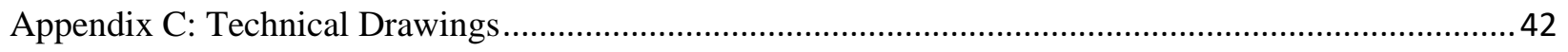




\title{
Nomenclature
}

E-Elastic Modulus

$S_{y}-$ Yield Strength

TS - Tensile Strength

$U_{r}-$ Modulus of Resilience

\section{Greek Letters}

$$
\begin{aligned}
& v-\text { Poission's Ratio }^{\prime} \\
& \sigma_{y}-\text { Yield Stress }
\end{aligned}
$$

\section{List of Abbreviations and Acronyms}

\author{
AOA - Angle of Attack \\ CAD - Computer Aided Design \\ DOF - Degrees of Freedom \\ EAP - Electroactive Polymer \\ FFF - Fused Filament Fabrication \\ NACA - National Advisory Committee for Aeronautics \\ PETG - Polyethylene Terephthalate \\ PLA - Polylactic Acid \\ SST - Shear Stress Transport \\ STP - Standard Temperature and Pressure \\ TPU - Thermoplatic Polyurethane \\ Voxel-Volumetric Pixel
}




\section{List of Figures}

Figure 1: Wright Brother's Wing Warping Concept [1] …............................................................

Figure 2: Fowler Flaps Deployed on a Boeing 747 [2] .................................................................. 2

Figure 3: Impact of Mesh Sizing on Surface Resolution [3] .............................................................

Figure 4: EAP Geometry Change due to Applied Voltage [4] .......................................................... 4

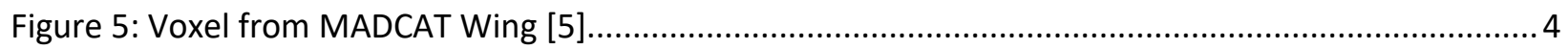

Figure 6: Voxel Assembly with Hexagonal Skin Panels [5] ................................................................. 4

Figure 7: FlexSys Wing Cross-Section [7] ............................................................................... 5

Figure 8: FlexSys' Actuators and Compliant Structure [7] ............................................................. 5

Figure 9: ETH Zurich Corrugated Structure Morphing Airfoil [8]...................................................... 6

Figure 10: University of West England Morphing Wing [9] .......................................................... 6

Figure 11: Example of Skin Buckling on Compressed Surface [11] ................................................... 8

Figure 12: Example of Biomimicry in the shape of the Shinkansen Bullet Train [14]............................ 9

Figure 13: X-Ray Image of a Fish Skeleton [19] .......................................................................... 12

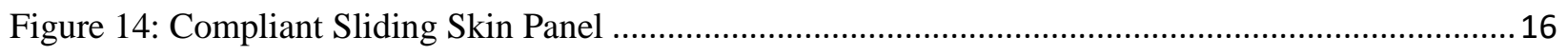

Figure 15: Oblique View 1 of V4 Assembly without Skin Panels ...................................................... 17

Figure 16: Oblique View 2 of V4 Assembly without Skin Panels ...................................................... 17

Figure 17: Prototype in Neutral Configuration .............................................................................. 19

Figure 18: Prototype in Maximum Deflection Configuration............................................................ 19

Figure 19: FEA Model with Zero Degrees Trailing Edge Deflection....................................................2

Figure 20: FEA Model with Ten Degrees Trailing Edge Deflection ......................................................21

Figure 21: FEA Model with Twenty Degrees Trailing Edge Deflection ...............................................2

Figure 22: FEA Model with Thirty Degrees Trailing Edge Deflection .................................................21

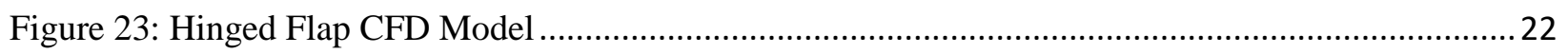

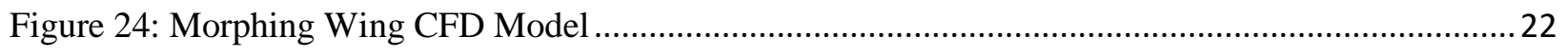

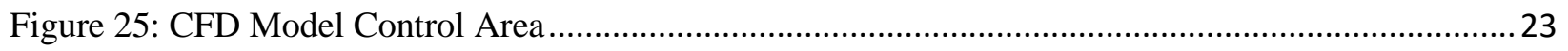

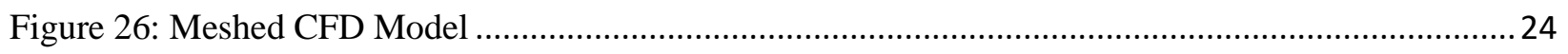

Figure 27: Detailed CFD Mesh View of Thirty Degree Morphed Wing ...............................................24

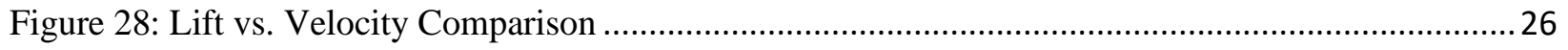

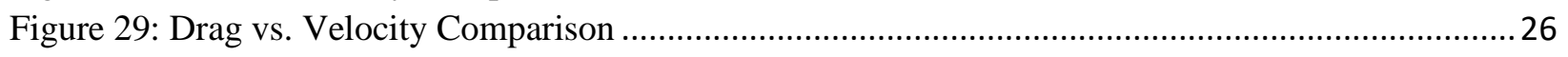

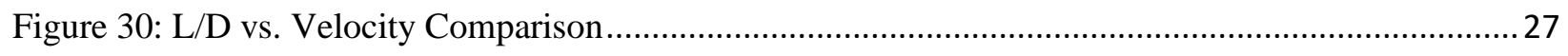

Figure 31: Deflection Angle vs. Lift Coefficient Comparison .............................................................. 27

Figure 32: 0 deg Hinged Flap Pressure Contour.............................................................................. 34

Figure 33: 0 deg Hinged Flap Velocity Contour.............................................................................. 34

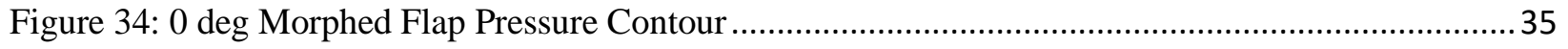

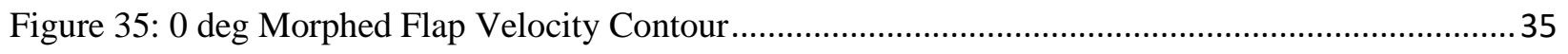

Figure 36: 10 deg Hinged Flap Pressure Contour.............................................................................. 36

Figure 37: 10 deg Hinged Flap Velocity Contour............................................................................... 36

Figure 38: 10 deg Morphed Flap Pressure Contour .......................................................................... 37

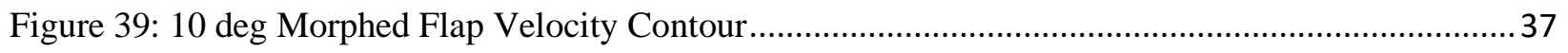

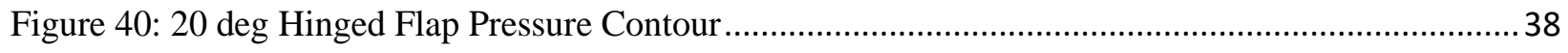

Figure 41: 20 deg Hinged Flap Velocity Contour.......................................................................... 38 
Figure 42: 20 deg Morphed Flap Pressure Contour... 39

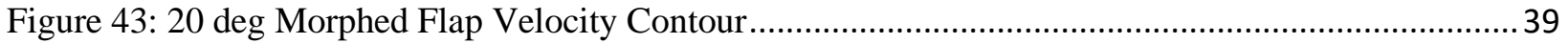

Figure 44: 30 deg Hinged Flap Pressure Contour........................................................................... 40

Figure 45: 30 deg Hinged Flap Velocity Contour................................................................................ 40

Figure 46: 30 deg Morphed Flap Pressure Contour .......................................................................... 41

Figure 47: 30 deg Morphed Flap Velocity Contour............................................................................ 41

\section{List of Tables}

Table 1: Yield Strength over Young's Modulus and Modulus of Resilience Values [15] ........................10

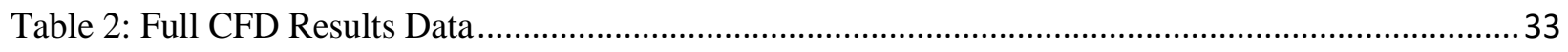




\subsection{Introduction}

As long as man has been able to achieve flight, they have been pushing the boundary of what is possible in both air and space. This has been done through tireless research and development in the search of ever-increasing performance. One fundamental principle of flight is that the lift generated must exceed the weight of the aircraft to takeoff. Once the aircraft is in the air, the changes in pressure between the upper and lower surface of the wing could be manipulated to control the roll, pitch and yaw of the plane.

The method of high lift generation and orientation of the aircraft has become widely accepted to be controlled through discrete elements such as flaps, ailerons, rudders, etc. This has become the widely accepted solution but has not always been the case. The Wright brothers, who achieved the first-ever successful powered flight, did not use the aforementioned discrete control surfaces. Instead they implemented something that was called wing warping. Wing warping worked by twisting the wing so that the angle of attack would vary across the span of the wing, with one side experiencing an increase, while the other experienced a decrease. This change in angle of attack allowed for control over the lift generated on each side and therefore allowed for control over the lateral stability of the airplane [1].

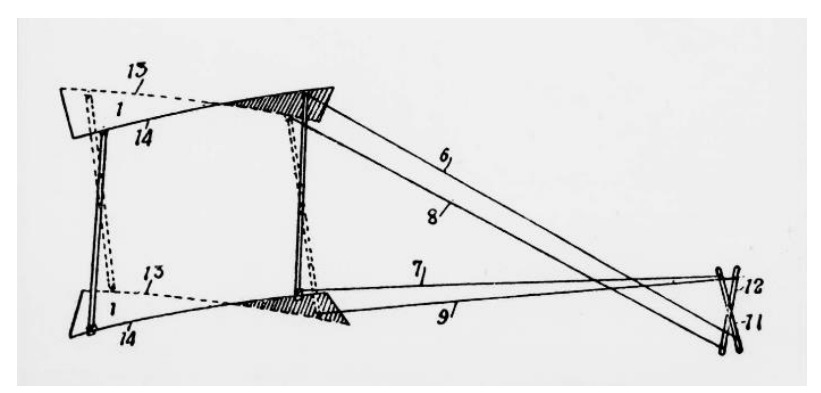

Figure 1: Wright Brother's Wing Warping Concept [1]

As mentioned previously, control surfaces today are made of discrete elements that commonly feature hinged joints and sliding mechanisms. One of the most common types of flaps used in aircraft today are slotted flaps, which can be seen on aircraft of all sizes. These flaps serve to increase the wing camber as well as create a slot between the main wing and flap for high energy air to be added to the boundary layer along the upper surface. This allows the air to stay attached longer, allowing for higher angles of attack on the flaps and therefore, higher amounts of lift. On large aircraft, such as the 747 which can be seen below, use a flap-type called the Fowler Flap. This type of flap incorporates translation and rotation of the flap elements. In the case of the 747, multiple discrete elements all track and rotate from the main body of the wing. This creates several slots in which high energy air can be added to the upper surface boundary layer. This allows for large amounts of lift generation at low airspeeds without stalling the aircraft. 


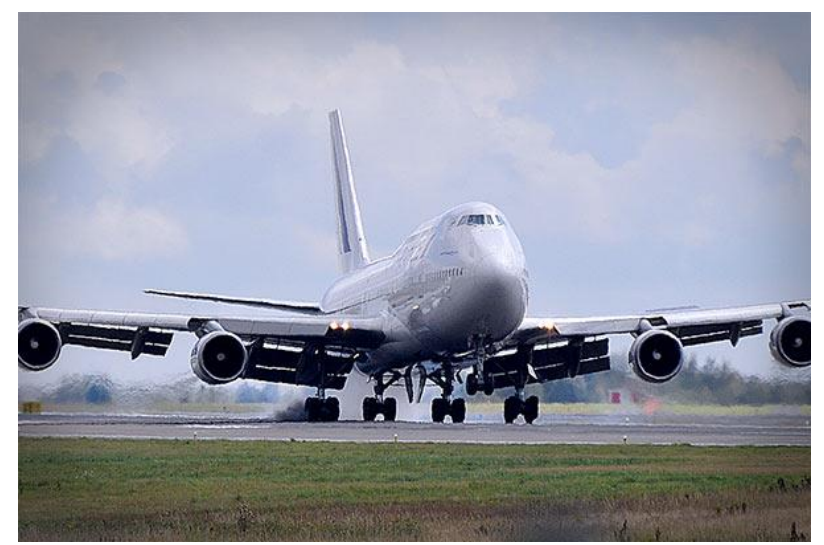

Figure 2: Fowler Flaps Deployed on a Boeing 747 [2]

This report will serve as an exploratory look into new methods and ideas by which a morphing wing could be designed. The goal of these designs would be to increase the lift and efficiency with which lift is generated. These designs also carry a goal of being as simple as possible, so that any factors such as weight, part count, manufacturing, or maintenance would not single-handedly act as the Achilles heel to its feasibility.

This report is structured in a way that first reviews existing technologies. These are technologies being developed by research groups at universities as well as conceptual technologies being marketed by companies. Trends are established to be investigated later in the report. Next, the preliminary approach is discussed and the methodologies that served as an inspiration for this project. Following the preliminary analysis, an overview is provided of all five design iterations that were designed, 3-D printed, and tested for this project, with two designs being selected as one's worth pursuing further. The design iteration section includes some testing and lessons learned from skin designs that were found during the preliminary research stages. These lessons learned were applied in the following section where a new type of skin panel structure was designed to work with the chosen rib models. Following the research and design sections of the report, a small section was completed to explain the work done to take the simple SolidWorks single rib designs and adapt it to be a section of wing comprised of ribs, spars, and complete skin panels. The analysis section followed and covers the finite element and computational fluid dynamics work done to gauge the performance of the chosen design. The computational fluid dynamics section also conducts the same analysis on the same base airfoil with a hinged flap design. Finally, the main body of the report ends with a discussion and presentation of the results of the CFD analysis, with final conclusions drawn based on the results. 


\subsection{Review of Existing Technologies}

To begin the process of designing and developing morphing wing technologies, a preliminary review of existing technologies and concepts must be completed. Several examples of concepts and preliminary implementations of these technologies were found, and their underlying materials and structures were analyzed. One of the first examples of this technology that was found was implemented into the automotive industry as a BMW concept car called the BMW Next 100. The BMW appears to be quite simple in both its form and function. The BMW uses rigid triangular-shaped plates, which allow them to interlock around varying curvatures and geometries. By decreasing the size of the triangular tiles, the plates will be able to match ever-increasingly complex geometries (see meshing image below). The plates are suspended on an elastomeric skin. This means that the skin will exhibit rubber-like properties, with the ability to stretch and deform, while maintaining the ability to return to its original shape. The elastomer skin will be suspended from the surrounding wheel arch, over a non-rotating portion of the outside surface of the wheel. By suspending between these surfaces, the skin is free to stretch and release as the driver turns the steering wheel to change the angle of the front tires.

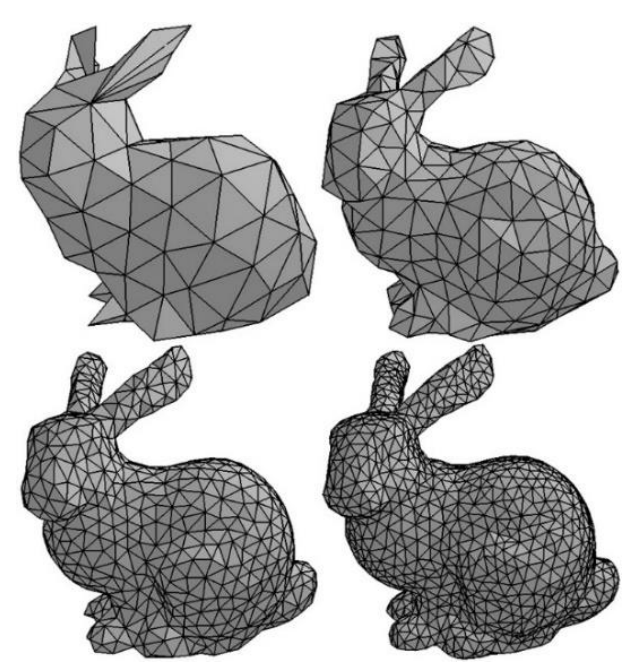

Figure 3: Impact of Mesh Sizing on Surface Resolution [3]

Another example found in the automotive industry was the Hankook Hexonic tire. This tire is similar to the Next 100 because it uses interlocking tiles suspended on an elastomer skin. The difference in the Hankook, is that the tiles are a standard synthetic rubber traditionally used in tire manufacturing. Additionally, the elastomer used in the tire for the skin between the rubber tiles is an electroactive polymer (EAP) [4]. EAP's are a polymer that can exhibit changes in shape and geometry when a voltage is applied. As shown in the image below, when a voltage is applied the elastomer is compressed and stretched, generating an outward expanding force. In the case of the Hankook tire, this activation is what creates the expanding channels to help the tire clear water and adapt to changing weather conditions [4]. 

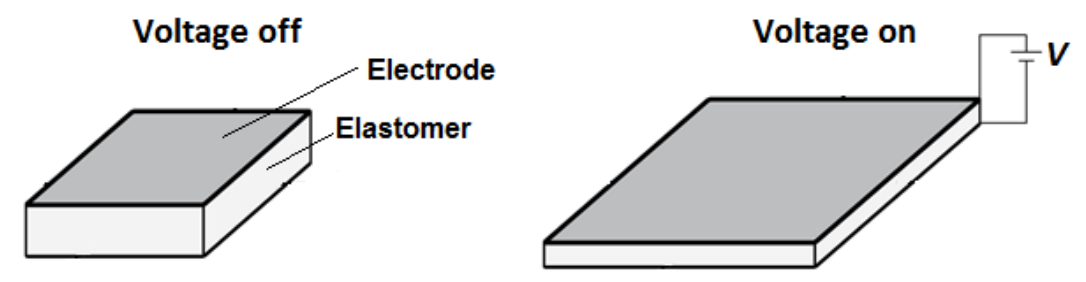

Figure 4: EAP Geometry Change due to Applied Voltage [4]

The first morphing surface technology that was found in the aerospace industry was the MADCAT morphing wing, which is a collaborative project between NASA and MIT. This concept is comprised of octahedral blocks arranged in a lattice pattern referred to as voxels (volumetric pixels), which can be seen below. Just like how two-dimensional images are made up of an arrangement of pixels, a threedimensional geometry can be comprised of an arrangement of voxels [5]. The voxels fit together like Lego bricks, which allow them to be easily rearranged [5]. The surface level voxels are covered in hexagonal tiles that interlock with the surrounding tiles. By rearranging the voxels, the surface geometry can be altered to maximize efficiency in any flight condition.

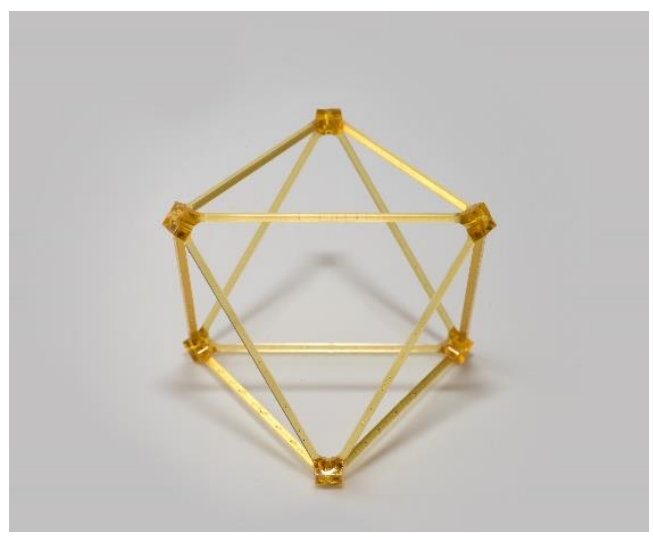

Figure 5: Voxel from MADCAT Wing [5]
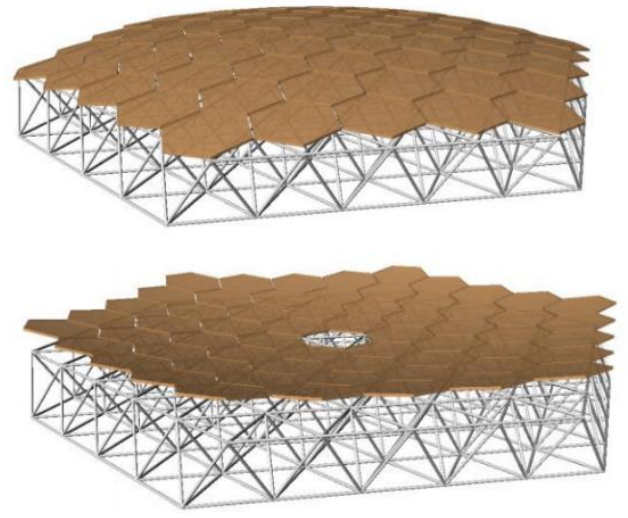

Figure 6: Voxel Assembly with Hexagonal Skin Panels [5]

Another aerospace-related morphing surface technology that has thoroughly developed its system is the morphing wing by FlexSys. They are one of the world's leading developers of morphing wing technology. As seen in Figure 5, the Flexfoil is divided into three main sections; the flexible leading edge, the rigid center portion, and the flexible trailing edge. Each of these portions appear to make up approximately one-third of the chord length. The structure of the flexible portions of the wing is made up 
of one-piece compliant mechanisms, which allow for a jointless-mechanism that more evenly distributes the load throughout the entire structure [6].

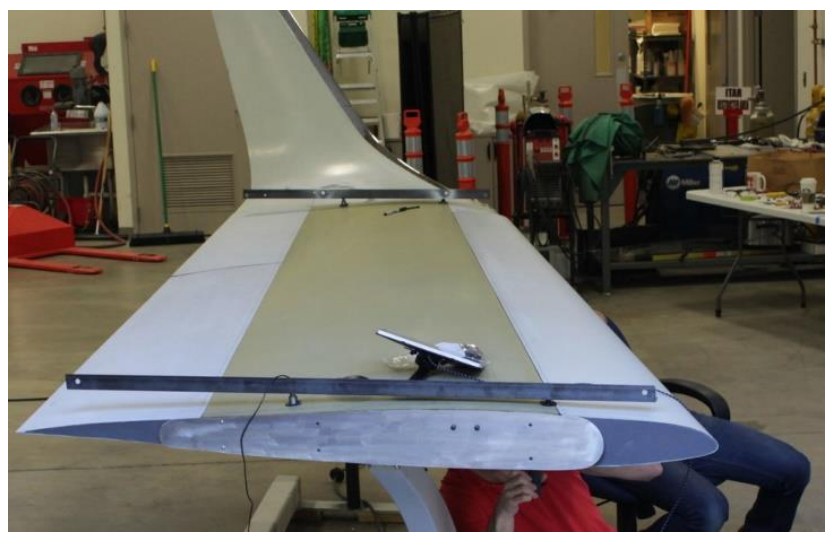

Figure 7: FlexSys Wing Cross-Section [7]

It can be seen in the above figure that there is a seam where the flexible portions meet the rigid middle section. In Figure 6, the actuation system of the airfoil can be seen. The actual morphing of the airfoil is controlled by servo motors which generate the linear motion to deflect the leading and trailing edges. The skin appears to be riveted to the rigid portion and pre-tensioned across the inner structure of the morphing segments to prevent buckling of the skin under deformation. The skin material most likely changes across this seam, with the center section of the wing being covered in a traditional airplane skin material. It was also found that the flexible portions of the Flexfoil are covered with an elastomeric composite skin [7].

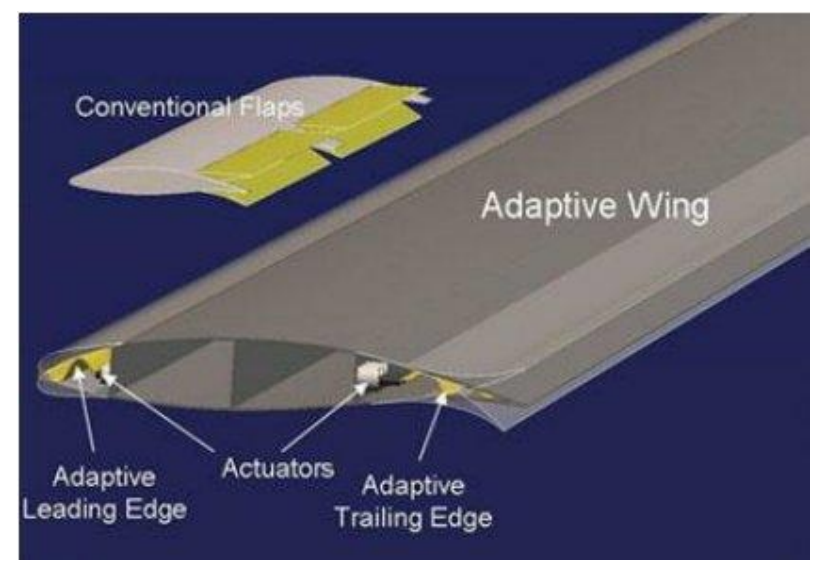

Figure 8: FlexSys' Actuators and Compliant Structure [7] 
Morphing wings are still very much in the research and development stage. With much of that research being conducted by Universities. One such example can be found at ETH Zurich. Their solution uses a corrugated structure in the aft portion of the airfoil. The advent of a corrugated structure is that it provides anisotropic mechanical properties. In the case of the ETH morphing wing, the in-plane stiffness is very high, while the out-of-plane stiffness is relatively low. This allows the airfoil to bend and flex with relatively low actuation force but remains rigid under aerodynamic loading. The ETH wing however has a discontinuous lower surface due to the corrugation and without an appropriate applied skin, it appears there are little aerodynamic benefits.

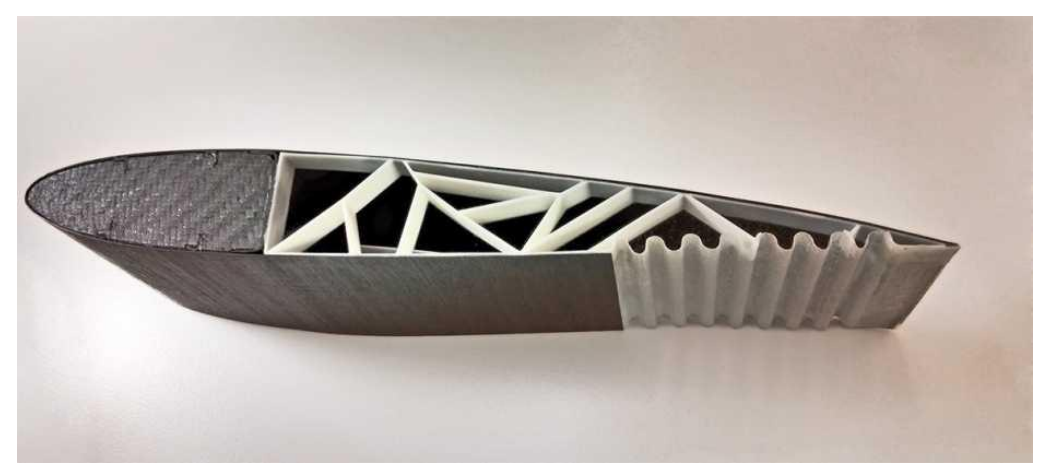

Figure 9: ETH Zurich Corrugated Structure Morphing Airfoil [8]

In the paper "Further Development of a Variable Camber Morphing Mechanism Using the Direct Control Airfoil Geometry Concept", an emphasis is placed on the importance of skin material selection and design. They research new methods of skin attachment and skin selection through tensile and fatigue testing. The research conducted in this paper borrows heavily from the FishBAC concept created at the University of Bristol. Both concepts use "a skeleton-like structure to impose a smooth continuous change in airfoil camber" [9]. Similarly, to other morphing wing designs, the attachment points of the skin to the corrugated structure bear much of the load.

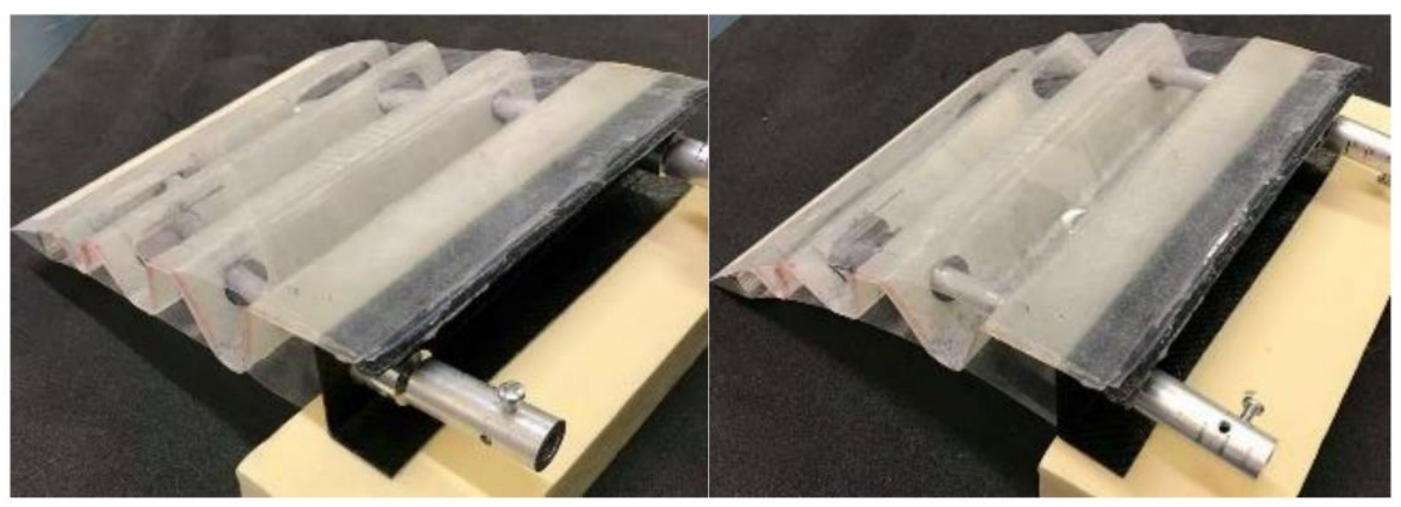

Figure 10: University of West England Morphing Wing [9] 
Based on the research conducted, they determined that an elastomer would be the optimal skin material choice to achieve a flap deflection of +25 degrees [9]. They conducted tensile and fatigue tests on silicone sheets with thicknesses of $0.5 \mathrm{~mm}$ and $1 \mathrm{~mm}$ and varying hardness' of $30-80$ shore. The conclusion was reached that a thickness of $0.5 \mathrm{~mm}$ with a hardness of 40 shore was the optimal skin choice due to it having the lowest required actuation force [9]. Another key point of this paper by the University of West England was to determine a more optimal method of attachment between the skin and the corrugated structure underneath. The final method they came up with involved pre-tensioning the skin to eliminate buckling as previously discussed. Then they attached the skin via a silicone glue called Elastosil E41 [9]. The results showed a bidirectional flap deflection of 23 degrees without the skin. Once the skin was attached the downwards and upwards deflections became 15.26 degrees and 10.27 degrees respectively [9].

The article titled "Skin design studies for variable camber morphing airfoils" provides further deep insights into the design, selection, and testing of various skin materials for morphing wings. In the article they state that the general requirements for a morphing wing skin would be high strain capabilities, with moderate to low stiffness in order to decrease actuation force requirements [10]. This article makes note of a project by NextGen Aeronautics, who developed a wing that could change its aspect ratio by $200 \%$, area by $70 \%$ and span by $40 \%$ [10]. In said project, an elastomeric silicone material was chosen for the skin. The NextGen airfoil also used a metallic ribbon structure to provide large out-of-plane stiffness to remain rigid under aerodynamic loads [10]. Both the structural and material choices appear to be very consistent across most of the research that was reviewed and appear to be the current optimal choices.

This article concludes that the optimal skin for a morphing wing would be made of a highly anisotropic material, with low axial stiffness to allow the airfoil to adjust its camber using relatively low actuation force [10]. It also states that there is a distinct limit below which the axial stiffness should not decreased. This would cause "unacceptable global camber deformation under the external aerodynamic loads" [10].

The research done in the paper "Development of variable camber wing with morphing leading and trailing sections using corrugated structures" echoes many of the ideas found in previous papers. One distinct difference in their paper was the use of different materials for the upper and lower surface morphing skin. The upper surface of their prototype used carbon fiber reinforced plastic (CFRP) laminates and 0.2 $\mathrm{mm}$ thick polypropylene sheets for the lower surface [11]. Other noteworthy points from their research was that a downward morphing caused buckling of the polypropylene lower skin. They stated that some proposed ways to solve this would be pre-tensioning the skin or adding a sliding mechanism into the lower skin [11]. This induced skin buckling can be seen in the figure below.

Other important criteria to consider when analyzing the impact of morphing wings and their skins, are reactions to aerodynamic loads. Under high aerodynamic loads, the aeroelasticity and inertia of the structures to which the loads are applied can oscillate violently in a phenomenon called "flutter". This topic of flutter in morphing wings in analyzed in the paper "Flutter Analysis of a Morphing Wing Technology Demonstrator: Numerical Simulation and Wind Tunnel Testing" [12]. They perform analysis and comparison between a traditional $3 \mathrm{~mm}$ aluminum skin panel and an optimized carbon fiber composite skin panel. The aluminum is kept at a constant thickness throughout the panel, whereas, the carbon fiber composite panel has varying plies and ply thicknesses. 


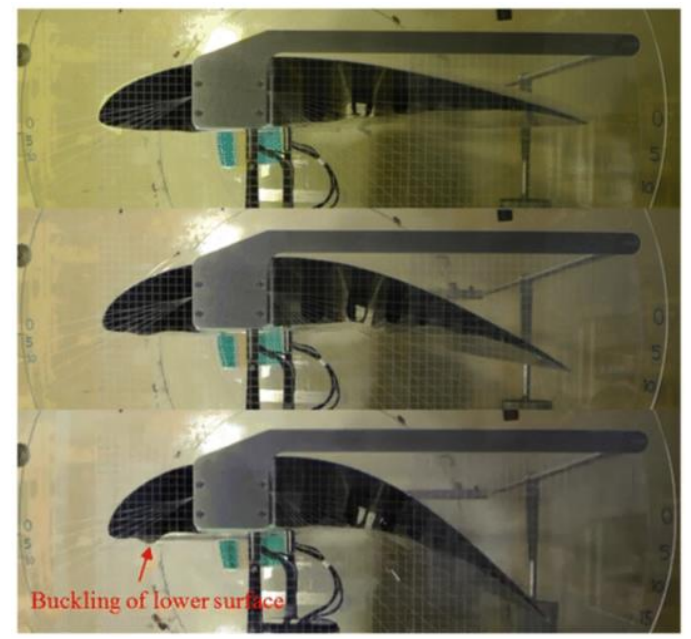

Figure 11: Example of Skin Buckling on Compressed Surface [11]

In their morphing wing design the varying plies and ply thicknesses were used to tune the aeroelasticity across the wing [12]. The panels of carbon fiber composite morphing skin were fixed at their ends to the spar caps to maintain a continuous curvature and tangency under deformation. Their overall wing design was made up of six total skin panels; two for the wing box, two for the trailing edge, and one for the leading edge [12].

The research conducted lead to very interesting results when the morphing skin is directly compared to the traditional skin. The testing concluded that the natural frequencies of the morphing and traditional skin were identical. Additionally, stiffness testing found that the spanwise stiffness of the morphing wing was greater. The chordwise stiffness of the morphing wing was lower, as to allow for the actual morphing to take place [12]. Another interesting conclusion made from the research was that the upper skin material has no impact on the torsional stiffness of the wing as the two materials performed identically [12]. The paper concludes that the two materials behaved very similarly in all tests, with the only notable differences in span-wise and chord-wise stiffness as previously mentioned [12]. The carbon fiber material was optimized to perform as well as the traditional aluminum skin and the results demonstrated that the composite skin had "minimal influence on the aeroelastic behavior of the wing" [12].

Based on the technology and literature review done, there were a few key takeaways. Firstly, the use of an elastomer skin appears to be acceptable, with silicone materials being the most favored. To avoid the elastomer skin buckling on the compressed surface of the wing, most examples pre-tension the skin. The structure should be anisotropic to allow for minimal required actuation force in the desired direction yet remain stiff enough to endure aerodynamic loads without buckling. An optimally designed skin should have negligible impact on the aeroelastic characteristics when compared to a traditional wing skin. Finally, as most of the concepts and technologies that were reviewed are based on a camber morphing design, it should be understood that these conclusions on ideal properties could change if the goal was to morph other wing characteristics such as sweep, chord, span, etc. 


\subsection{Inspiration and Preliminary Approach}

The initial inspiration for the design of the morphing wing came from an attempt to implement biomimicry into the design as much as possible. A real-world example of implementations of biomimicry can be seen in the figure below. In Japan, sonic booms were being created by the Shinkansen (bullet train) when it exited tunnels. To mitigate the sonic booms, they modeled the nose of the train after the Kingfisher. The Kingfisher is known for diving into the water to catch its prey, all while making a minimal splash upon breaking the surface of the water. By mimicking its beak shape into the nose of the train, the redesigned train was able to achieve $10 \%$ higher speeds, was $15 \%$ more efficient, and kept the train below the $70 \mathrm{~dB}$ noise limit through residential areas [13].

Research was done into the skeletal structures of both birds and fish. Additionally, the skin materials of scales and feathers were researched to see the feasibility of their implementation. These skin materials were also used to investigate the ability to maintain a smooth contour given small discrete elements. The motion of structures in nature rarely see the implementation of something such as a pin joint, rather it will flex and comply with applied loads to move. This approach was also taken with the design of this morphing wing in the form of a compliant mechanism being used for the wing rib structure and skin panels. The hypothesis is that this approach allows the entire wing cross-section to change its shape to maximize efficiency in all stages of flight.

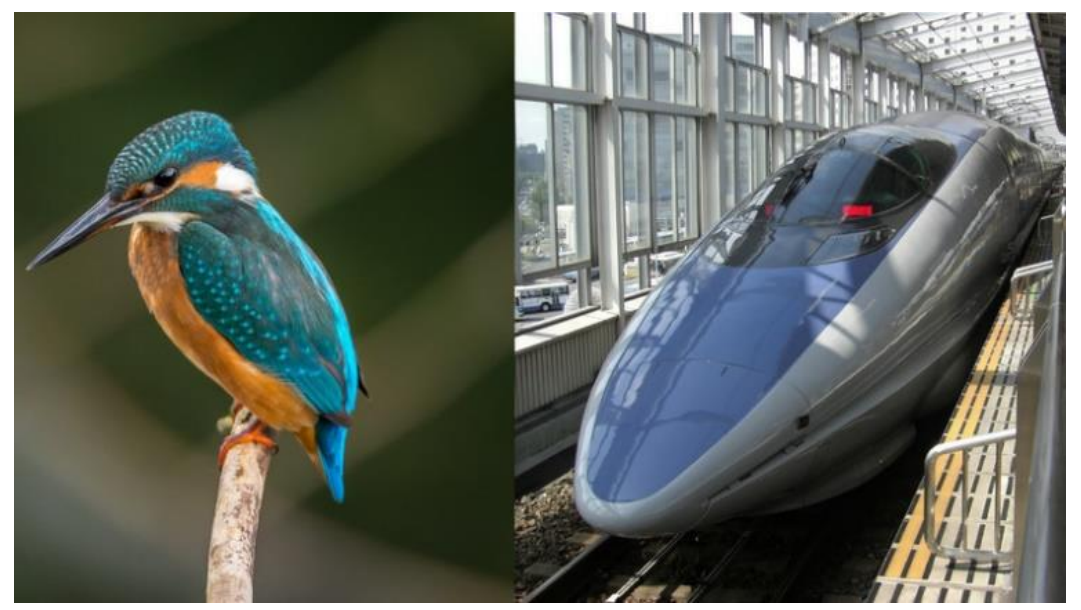

Figure 12: Example of Biomimicry in the shape of the Shinkansen Bullet Train [14]

The first test done for the design of the morphing wing was to test the feasibility of using a desktop FFF 3D printer and readily available materials to create such compliant mechanisms. Several compliant 4bar mechanisms were printed with varying flexure widths from $0.6-2 \mathrm{~mm}$ at $0.2 \mathrm{~mm}$ intervals. The materials used were three of the most commonly found FFF materials; PLA (Polylactic Acid), PETG (Polyethylene terephthalate), and TPU (Thermoplastic Polyurethane). For this initial test, only qualitative results were taken. It was found that the PLA required the most actuation force, followed by PETG and finally TPU. The TPU, which has the lowest stiffness of the materials tested was able to endure angular displacements 
on the flexures of approximately $\pm 90^{\circ}$ without any plastic deformation or signs of fatigue after approximately 500 cycles. Comparatively, both the PETG and PLA failed at the flexures when attempting displacements of approximately $\pm 45^{\circ}$, with the PLA breaking after fewer cycles than the PETG. As expected, the required actuation force increased with the flexure width, but had little impact on the number of cycles before failure when the flexure width was between 0.8 and $1.6 \mathrm{~mm}$. The iterations that performed the best in terms of cycles before failures were once in which the flexure width matched a factor of the nozzle diameter of the printer. By matching the flexure width to a factor of the nozzle width, a continuous layer was created across the flexure without having to adjust the extrusion multiplier. This had the advent of creating a uniform and continuous load path to distribute the stress across the flexure. The printer used had a $0.4 \mathrm{~mm}$ nozzle, so the best performers of those tested were the $0.8,1.2$, and $1.6 \mathrm{~mm}$ variants.

Based on properties discussed in Handbook of Compliant Mechanisms by Larry Howell, the ideal material for a compliant mechanism is one that is both strong and flexible. The metric used to deduce this is the ratio between the yield strength to the Young's Modulus, with a higher ratio being favorable [15]. Another metric used to rank materials for use in compliant mechanisms is its modulus of resilience. The modulus of resilience is a measure of how much energy per unit volume is required to create a permanent change in the material [15]. The equation for the modulus of resilience as well as a table from Handbook of Compliant Mechanisms outlining common materials and their respective values can be seen below [15]:

$$
U_{r}=\frac{\sigma_{y}^{2}}{2 E}
$$

Table 1: Yield Strength over Young's Modulus and Modulus of Resilience Values [15]

\begin{tabular}{lcccc} 
& & & $\left(S_{y} / E\right) \times$ & $\left(0.5 \times S_{y}^{2} / E\right) \times$ \\
Material & $E(\mathrm{GPa})$ & $S_{y}(\mathrm{MPa})$ & 1000 & 0.001 \\
\hline Steel (1010 hot rolled) & 207 & 179 & 0.87 & 77 \\
Steel (4140 Q\&T @400) & 207 & 1641 & 7.9 & 6500 \\
Aluminum (110 annealed) & 71.7 & 34 & 0.48 & 8.1 \\
Aluminum (7075 heat treated) & 71.7 & 503 & 7.0 & 1800 \\
Titanium (Ti-35A annealed) & 114 & 207 & 1.8 & 190 \\
Titanium (Ti-13 heat treated) & 114 & 1170 & 10 & 6000 \\
Nitinol (high-temperature phase) & 75 & 560 & 7.5 & 2100 \\
Beryllium copper (CA170) & 128 & 1170 & 9.2 & 5300 \\
Polycrystalline silicon & 169 & 930 & 5.5 & 2600 \\
Polyethylene (HDPE) & 1.4 & 28 & 20 & 280 \\
Nylon (type 66) & 2.8 & 55 & 20 & 540 \\
Polypropylene & 1.4 & 34 & 25 & 410 \\
Kevlar (82 vol\%) in epoxy & 86 & 1517 & 18 & 13000 \\
E-glass (73.3 vol\%) in epoxy & 56 & 1640 & 29 & 24000 \\
\hline
\end{tabular}

Based on the above equation, the values for PLA, PETG, and TPU are respectively are: $250 \times 10^{3}$ [16], $420 \times 10^{3}$ [17], and $800 \times 10^{3}$ [18]. PLA had the lowest resilience with PETG's resilience being approximately $40 \%$ better and TPU approximately $69 \%$ better. Therefore, for the remaining tests PLA was removed as a feasible material for the development of the compliant wing rib structure. Additionally, it 
became clear through this test that TPU could be used for extremely large deflection designs of wing ribs but PETG would require several flexural points whose angular displacements would add together to become larger deflections. These ideas of less flexure with higher displacements versus several flexures with lower displacements are discussed and tested in the following section. Regardless of the method taken for the structure the desired design of the wing should maintain a continuous curve along the chord as opposed to a single large displacement point that just behaves in a similar manner to a pin joint on which an aileron or flap is traditionally attached. With the lessons learned in these tests, it appears completely feasible to apply additive manufacturing techniques such as FFF to create compliant mechanisms as internal wing structures.

\subsection{Wing Rib Design Iterations}

The objective with all the wing rib designs was to observe a smooth change in camber as the actuation load was applied and to maintain a continuous curve along the chord of the rib to which the skin could later be applied. For all the wing rib designs created, a $200 \mathrm{~mm}$ chord section of a NACA 0018 airfoil was sectioned into thirds, with the leading third and most aft third being rigid structures. This left the center third of the chord section to house the compliant structure in which the camber morphing would take place.

\subsection{V1 - 4-Bar Compliant Mechanism}

The objective of the first design was to implement the knowledge gained from the initial 4-bar compliant mechanism tests directly into a wing rib structure to observe the change in geometry with an applied load. The leading and trailing edge segments of this design were printed in PETG to allow for a rigid structure. The compliant middle portion of the system was printed in TPU for its excellent resilience properties. This central portion was a 4-bar mechanism with the bottom link rigidly connected to the leading edge and the most aft link of the mechanism rigidly attached to the trailing edge. These boundary conditions allowed the system to have a trailing edge which could pivot downwards, behaving like a flap. However, this system merely emulated a pin joint that only allowed for motion in one direction. Additionally, by fixing the bottom link, the top link of the 4-bar mechanism translates and rotates with respect to the leadingedge potion. As a result, for the skin design, the chosen material would need to accommodate the expanding gap while maintaining structural integrity when stretched spanwise between the ribs. As for the curvature of the wing rib, this mechanism design allows for two chordwise flexural points at which an angular displacement can take place. It was observed from this test that two flexure sections will most likely not be sufficient to create a skin surface that appears to maintain a constant curvature. 


\subsection{V2 - 5-Bar Compliant Mechanism}

The main lesson learned from the V1 design was that the mechanism needs to be designed with an attachment that allows for symmetrical bending so that the rib structure could be used to alter the camber in either direction as seen in an aileron. The solution to this was to create an extra flexure at the midpoint of the forward link of the 4-bar mechanism to allow for symmetrical bending of the system. As in the V1 design, this system had the leading and trailing edge sections printed out of PETG and the compliant section was printed with TPU. The testing of this system showed that the design now allowed for symmetric bending to mimic the motion of an aileron. This design iteration incorporates an additional flexure which created three sections of the compliant mechanism that could now allow for rotation. However, this still did not appear sufficient as the skin would still need to expand and contract over large distances when going from +30 degrees to -30 degrees deflection. Upon the completion of the V2 iteration, little consideration for the skin had been taken. The goal for the early designs was to create a reliable and controllable complaint wing rib structure to provide a continuous curvature structure for camber morphing in the positive and negative directions. From the work completed up until this point it was clear that a change in design ethos was required in order to achieve the desired outcome.

\subsection{V3 - Compliant Multi-Segment Spine Using Flexible Material}

The initial goal of this project was to create a morphing wing design with an emphasis on biomimicry. Based on this, a new type of design was required to better incorporate this theme. For this design a close look was taken at the skeletal systems of fish to see what could be learned from the way they are able to contort their entire bodies in a way that resembles the camber morphing properties that are trying to be achieved through this project. An example of the skeletal system of a fish that was used for design inspiration can be seen below.

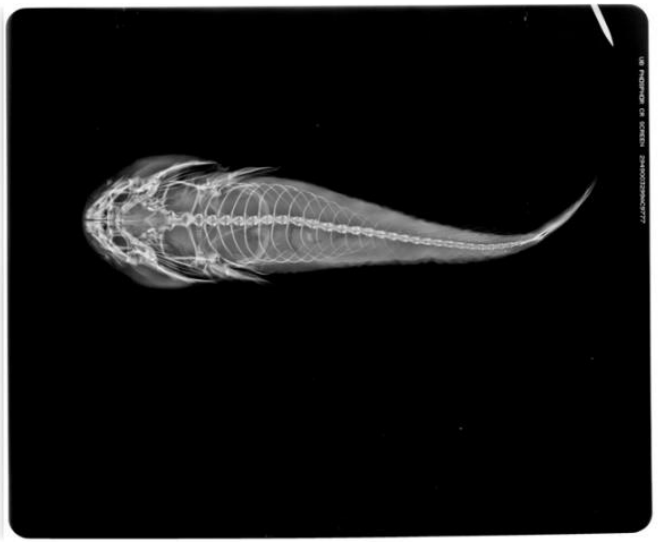

Figure 13: X-Ray Image of a Fish Skeleton [19] 
The result of this research was a rib design that maintained the rigid leading and trailing edge sections, but now incorporated a multi-segment spine which travels along the line of symmetry of the airfoil, connecting the two rigid sections. The spine, much like on the fish, allows for several pivoting segments along its length so that several small angular displacements can be made. The areas of small angular displacements arranged in series will create a large curvature when they are all combined along the chord length. The spine portion had material printed outwards to mimic the ribs that extend outwards from the spine of the fish. This material does not aid in the flexure of the spine but was used to create a support structure for the skin once it is attached.

Upon initial testing, the system proved to be a large improvement over the previous two iterations. The rib structures were printed in TPU with $1.2 \mathrm{~mm}$ flexures to make development easier as this material provided the highest resilience of the materials tested. Several tests were completed to compare the number of flexures with the continuity of the curvature of both the upper and lower surfaces. Each flexure section was designed so that each flexure could achieve an equal fraction of the required angular displacement (i.e. for 3 flexures, each must achieve $\pm 10^{\circ}$ for a total of the $\pm 30^{\circ}$ ). It was found through multiple iterations of printing that six flexures (each flexure provides $\pm 5^{\circ}$ ) appeared to be the optimal number. Iterations with fewer flexures created a more discrete looking surface on the compressed side. Iterations with a greater number of flexures provided diminishing returns as it became increasingly more difficult to manufacture while providing a smaller increase in the continuity of the compressed surface.

This design iteration also included the first attempt to incorporate an actuation system. Two ribs were printed along with a leading and trailing edge spar section that when combined created a small wing section of $200 \mathrm{~mm}$ chord and $100 \mathrm{~mm}$ span. The ribs were printed with a tubular section running chordwise along the upper and lower portions of the rib. These tubular sections acted as guides for control cables. The control cables were passed through the tubular sections and fixed to the trailing edge portion of the rib. On the leading-edge spar, a small 9-gram servo was mounted for each rib and the control cables were tied off on the servo horn. The servos were controlled via an Arduino Uno which took inputs from a linear potentiometer using ADC values and then sending PWM signals to the servos to match the relative rotational position of the potentiometer to the angle of the trailing edge of the wing section. The servos were synchronized by initially zeroing the servos to the same starting position. The next steps to improve on this system would be to include an encoder onto these servos so a feedback loop could be created to ensure even displacements across all ribs. Additionally, with a feedback loop incorporated into this system, spanwise aerodynamic twist could be controlled by evenly increasing or decreasing the camber at each rib.

\subsection{V4 - Compliant Multi-Segment Spine with Step-Down Skin}

The third iteration of this project was printed out of TPU, which as previously mentioned is a rubber-like material. This material choice would not be feasible for a final solution to be implemented in a plane of any scale. With the previous iteration serving as a proof of concept for the new flexure-based spine design, the goal of this iteration was to use a more rigid material to further converge on a design that could be feasibly implemented into a small-scale plane. Therefore, for this iteration, the ribs were printed in PETG.

To begin the adaptation for the V3 TPU based design to the V4 PETG based design, the first critical step was to determine the appropriate flexure size to maximize the lifecycle of the part while keeping the required actuation force low. This was determined by printing a series of flexures limited to an angular 
displacement of $\pm 5^{\circ}$. The flexures were printed from $0.4 \mathrm{~mm}$ to $1.6 \mathrm{~mm}$. It was found from the testing that the $0.4 \mathrm{~mm}$ and $0.6 \mathrm{~mm}$ flexures required almost no actuation force but broke very quickly due to the extremely thin connection across the flexure. The $0.8 \mathrm{~mm}$ to $1.2 \mathrm{~mm}$ flexures required a low actuation force and were able to withstand 500 cycles of manual testing without breaking or showing any major signs of fatigue. The $1.4 \mathrm{~mm}$ and $1.6 \mathrm{~mm}$ flexures also did not fracture or show any signs of fatigue, but it was found that the actuation force was noticeably higher. With the adjustments made from the V3 design, the new PETG V4 design was printed. Following the completion of the print the system was manually actuated through 500 cycles. The rib did not fracture, nor did it show any signs of fatigue.

This design iteration included the first major attempts to incorporate skin into the wing section design. From the preliminary research it was found that using an elastomer skin was a common choice. For the first test of the skin, two elastomer materials were selected; latex rubber and polyurethane fabric. In order to mitigate buckling on the side of the wing experiencing compression, the material was to be prestretched. In both cases the skin was attached to the rib using a high-strength adhesive. The skin material was attached by hand to the ribs, which was found to be very difficult. In order to apply the skin, the rib must be deployed to its maximum camber. The skin must then be stretched and applied to the compressed surface. The skin must be glued to each section of the rib individually and allowed to cure to ensure ample attachment force. It must be allowed to fully cure as this attachment point will then serve as an anchor point from which the skin is stretched to reach the next attachment point on the rib. It was found that even with pre-stretching the skin and allowing the adhesive to fully cure, small amounts of local buckling between the supporting sections of the rib occurred. Additionally, it was found that using these materials would add complexity as a rigid substrate would be required to support the skin in the spanwise direction between the ribs unless an appropriate anisotropic material could be found.

Experimentation on the use of a rigid skin was then initiated. The rib was redesigned so that it became a two-piece assembly that when assembled had a small internal channel on the upper and lower surface in which the skin could be attached. This internal channel would act as a guide for which the skin could be located and allowed to translate freely in the chordwise direction. The skin had a flange extending from the underside which was seated in the pocket of the rib. However, from testing it was found that based on the scale and resolution of the manufacturing methods used, the added height to the skin panel caused by the flange dramatically increased the stiffness of the panel. This increase in stiffness of the skin panel resulted in a large required actuation force to bend the skin. When the skin was tested in the rib, the rib quickly broke as the actuation force to change the camber caused too much stress in the flexures causing a fracture. To reduce the increased actuation force required, the flange on the skin was designed to mimic the structure that was already designed into the rib. The skin flange was sectioned to create flexures in the skin panel that complimented the rib. This allowed the flange, which is used for locating, to be supported in the pocket of the rib but allow the skin area that was self-supported to easily bend.

One immediate disadvantage of this design was that since the skin panel could translate to accommodate the extending and compressing surfaces of the wing, a gap is created by the moving skin. By fixing the skin panel to the rigid trailing edge section of the wing, this gap would be created at the leadingedge portion of the wing. To mitigate this, an overhanging section of stationary skin was added to the leading edge so that no gap in the skin was created. However, this meant that a step down was created from the leading-edge skin to the skin on the rest of the wing. This design was determined to be a viable direction for the final design. The impact of the step down in the skin will be tested and analyzed as described in the Computation Fluid Dynamics analysis section of the report to confirm the feasibility of this design. 


\subsection{V5 - Compliant Multi-Segment Spine with Split Trailing Edge}

For this fifth iteration on the wing rib design an emphasis was put on refining the work done on the V4 design, while trying to reduce the change in the skin surface such as the step-down. The solution that was created involved creating a split in the chordwise direction along the axis of symmetry on the rigid trailing edge portion. The skin could then be rigidly attached to both the leading and trailing edge sections of the rib. The center morphing section still contained the flanged skin with designed-in flexure points to keep the actuation force low. By rigidly attaching the skin to both the leading and trailing edge, a smooth surface could be created along much of the chord length of the wing. The caveat with this design is that the trailing edge split had to be incorporated so that the two halves of the trailing edge could act as a roller support to each other. The trailing edge section was constrained to allow translation using the other half of the trailing edge as a guide. This means that although much of the wing is made up of a continuous surface, there is a small change in angle at the trailing edge where the two trailing edge sections differ in position as the camber changes. It is worth noting though, that when the wing is in its uncambered position, a fully continuous surface exists, it is only when the camber is applied that a small portion of the trailing edge affects this continuous geometry.

\subsection{Compliant Skin Panel Design}

In previous sections where skin materials have been tested, the majority have been elastomers which were pre-stretched over the rib to mitigate buckling upon compression of either surface of the wing. This idea was what was found to be most common in industry conceptual designs as well as many research projects conducted by universities. From the testing that was done, this material choice proved to be very difficult to implement. The act of pre-stretching and adhering the skin to the rib in its tensioned state yielded inconsistent results in terms of the tension and contact area with the wing rib. Another issue that occurred, was even with pre-stretching, the material would fatigue over time and cause buckling to occur on the compressed surface. Additionally, from the tests with an elastomeric material, it didn't appear feasible for any scale of prototype as it posed even more challenges once an aerodynamic load would be applied. Therefore, it was decided, that such as with the wing rib, the skin should be designed as a compliant piece that would allow changes in camber, yet remain structurally integral under additional load conditions, such as when aerodynamic forces are added.

The V4 and V5 rib designs require the skin to slide along the wing rib structure, so as not to require compression and extension of the skin panels themselves. This means the skin panel could be made from traditional aircraft skin materials such as aluminum since the skin only need to accommodate chordwise bending for this camber morphing design. The skin would still need to be constrained against the wing rib in order to maintain the desired shape while in flight. So, for this skin design a small web and flange were added to the underside of the skin. This web and flange would act as a mechanism to consolidate a locking and sliding system to hold the skin firmly against the rib yet allow smooth chordwise motion. The skin panel was printed and tested using a panel thickness of $1 \mathrm{~mm}$ of PETG. Due to the resolution of the printer, the web and flange had to be printed larger than desired. The impact of this was the skin became too stiff for the required bending characteristics. This led to an increase of friction when tested inside a wing rib assembly and severely reduced the camber morphing capabilities. 
The solution to this problem was to mimic the design of the wing rib itself and implement deliberate stress concentrations in order to make a compliant skin panel structure. This was achieved through making $\mathrm{v}$-shaped cuts in the web and flange extending from the underside of the skin, which will be referred to as the skin rail throughout the rest of the report. This allowed a smooth increase in stress, but also served to create physical limitations the amount of bending that could be reached. An image of the skin panel design used in the prototype wing section can be seen below for reference. The v-cuts in the skin rail were placed in order to align and travel within the bounds between the rigid connections to the compliant section of the wing rib. This allowed for the lowest possible actuation force yet still provided easy translational motion along the surface of the wing rib.

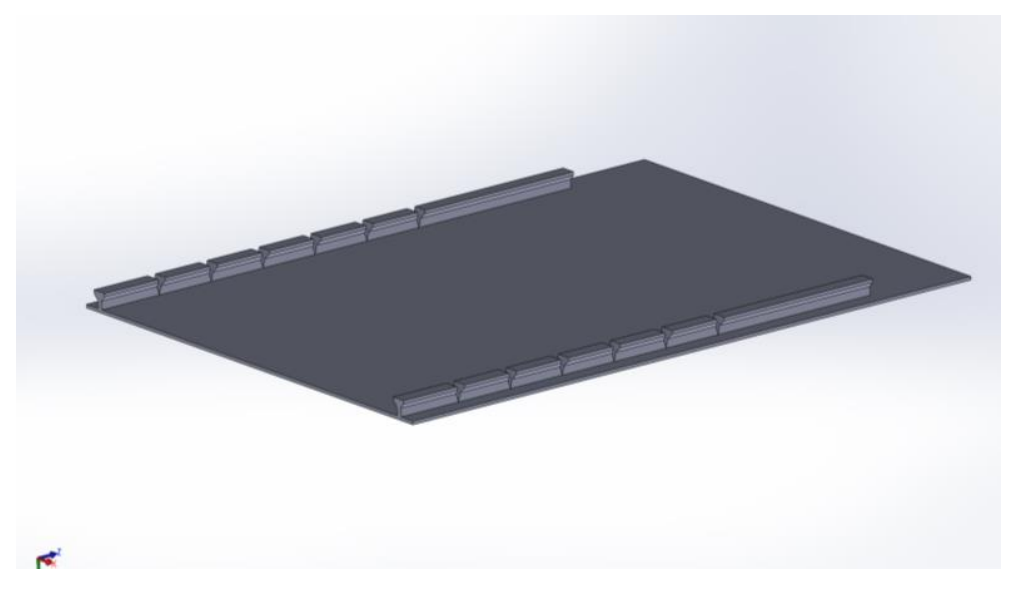

Figure 14: Compliant Sliding Skin Panel

\subsection{Morphing Wing Section Design}

In order to assess the capabilities of the design, a scale model was produced. It was decided that since the design created in the V4 iteration was simpler than the V5 design, the V4 should be tested first to assess its capabilities and whether a more complex design would be required. The model was prepared in SolidWorks and comprised of two rib assemblies, a leading-edge spar, a trailing edge spar, an upper and lower compliant skin panel, and a leading-edge skin panel. The wing section assembly was designed to have a chord of $200 \mathrm{~mm}$ and a span of $120 \mathrm{~mm}$ so all pieces could be easily printed on a mid-size desktop 3-D printer.

The leading-edge and trailing-edge spar were designed to be mounted to the rigid leading and trailing edge portions of the rib. This allowed for a simpler design as they would not need to comply with the changing camber of the small wing section. The spars were designed to incorporate the mounting of two 9-gram servos, which would serve as actuators for the manufactured prototype discussed in the following section. These spars were incorporated with the two-piece V4 wing rib assemblies, which allowed for the compliant skin panels to be easily attached to the model. Finally, the rigid leading-edge skin panel would fasten on the wing rib over the compliant skin in the rigid leading-edge portion of the rib. 


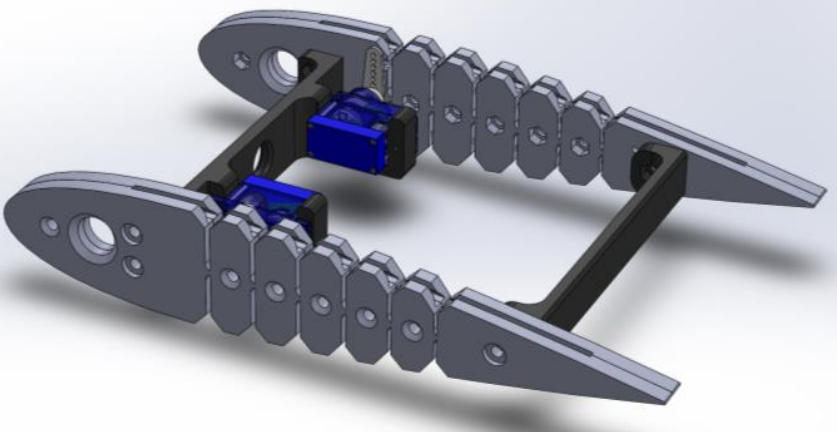

Figure 15: Oblique View 1 of V4 Assembly without Skin Panels

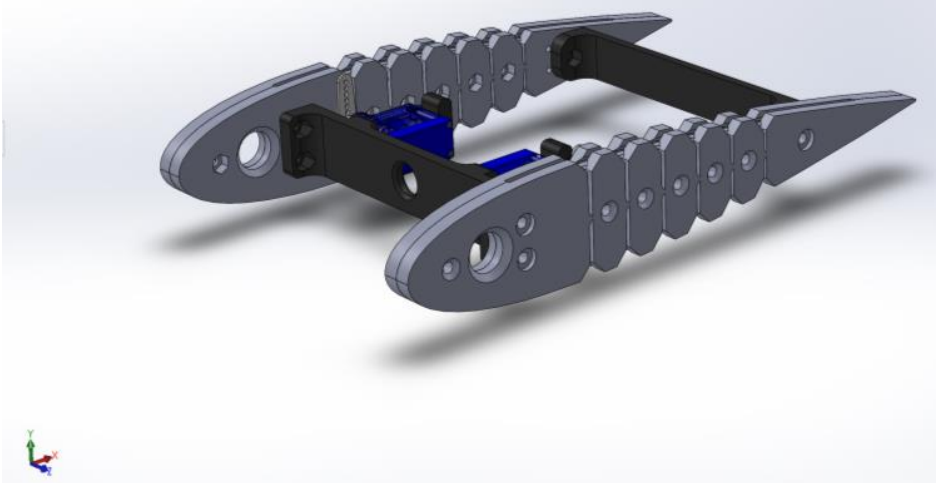

Figure 16: Oblique View 2 of V4 Assembly without Skin Panels 


\subsection{Manufactured Prototype}

The model designed and discussed in the previous section was specifically designed to be manufactured using 3-D printing techniques. The model was printed to understand how the system would behave once it is fully assembled. Additionally, it served as a check to ensure the design would survive the manual fatigue testing. Once this was completed, the design could pass onto the analysis stage.

Using 3-D printing allowed for a quick and low-cost small-scale prototype to be produced and tested. The material used for printing the prototype was the previously discussed PETG. As found in previous analysis, it was determined to be the best all-around performer for this initial test. The spars were printed as single pieces as the dimensions and complexity were tailored to work on a desktop 3-D printer. The ribs had to be printed as a two-piece assembly for two main reasons. Firstly, the channel along which the skin web and flange would travel created complex geometry that would have required support material. In most cases support material is not an issue, however it can lead to more difficult post-processing. In this model it would have been extremely difficult to remove all support material within the channel and ensure proper dimensional tolerancing. Secondly, the way the rib and skin were designed required that the skin be placed inside one side of the channel and then constrained when the other half of the rib assembly was fastened in place. This choice was made to speed up the design time and make printing and assembly easier.

The prototype was designed to house captive nuts as a threaded insert, meaning the whole assemble could be easily assembled and disassembled with a few screws. The skin was held in place due to the shape of the channel in the wing rib assembly and the skin rail. This meant the skin did not require any extra fasteners to be secured in place.

The individual parts were sliced in Cura to generate the G-code necessary for printing. As previously mentioned, the parts were all printed in PETG. The extruder and bed temperatures were set to 235 and 60 degrees Celsius respectively. The models were all printed with a $0.4 \mathrm{~mm}$ nozzle, at $0.2 \mathrm{~mm}$ layer heights. The bottom and top thicknesses were set to $1.2 \mathrm{~mm}$. The wall thickness was also set to 1.2 $\mathrm{mm}$ to ensure the flexures would be printed solidly with three complete passes of the nozzle at their thinnest point. Finally, all models were printed at $30 \%$ infill to keep the model rigid but keep the weight much below that of a solid part.

Once printing of the parts was completed, they were assembled. Following assembly, the model was tested to determine whether the tolerancing allowed for the skin panel to slide within the channel of the rib. The friction appeared quite low and morphing action was achieved with relative ease. Additionally, the flexures in the skin were placed to avoid binding between the rib structure and the skin rail. Testing revealed that the flexures had been placed effectively, with the bending force feeling quite low and no interference or binding occurring during deflection testing. The fatigue test conducted consisted of 1000 manual cycles of morphing between the maximum possible positive and negative deflection angles (approximately $\pm 30^{\circ}$ ). This test revealed little to no fatigue in the assembly. When released from a deflected state, the assembly would always return to its neutral state of $0^{\circ}$. Additionally, after the completion of the testing, each part was individually inspected. This inspection saw no damage, breakages, or signs of visible wear or fatigue. Two images have been included of the assembly; one of which in the neutral state, and the other in the state of maximum deflection. Technical drawings of all parts can be found in Appendix C. 


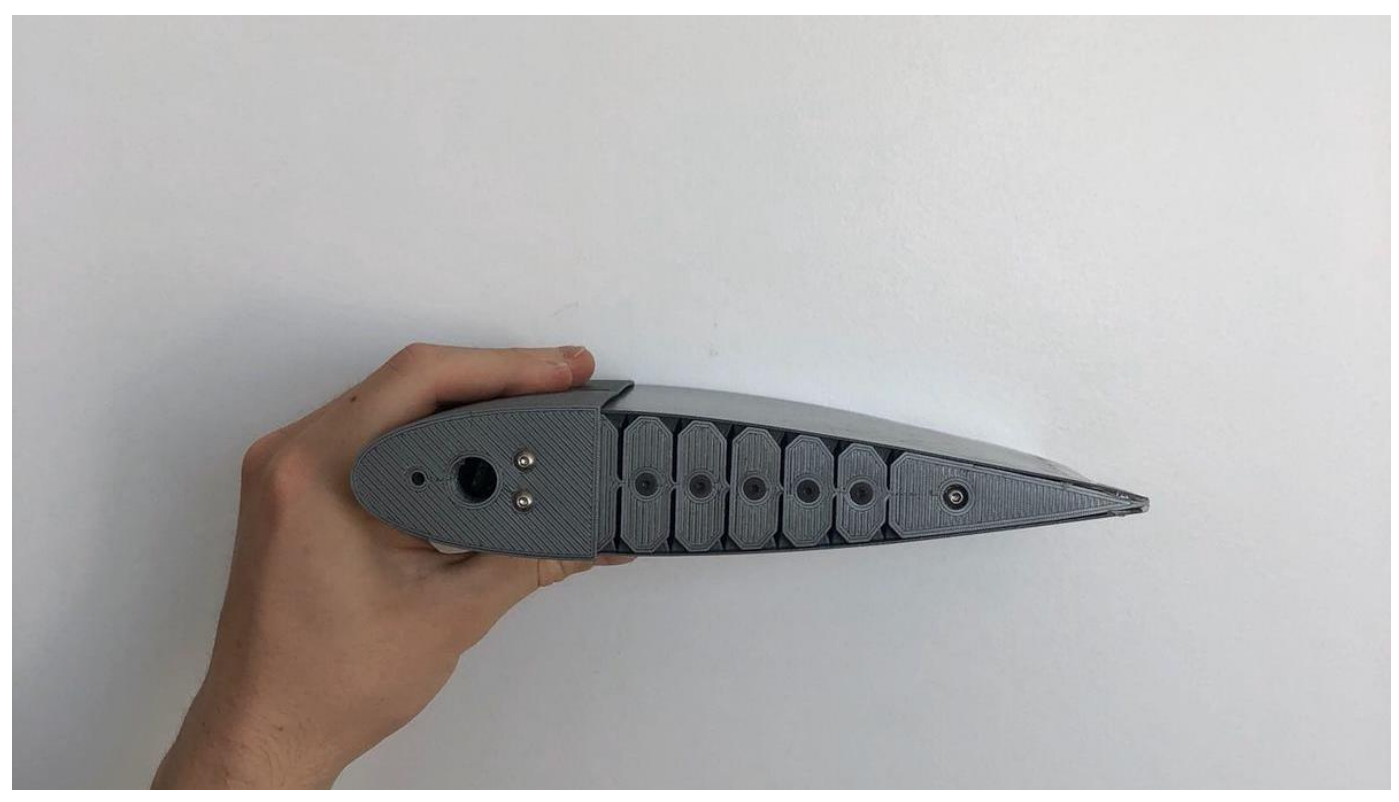

Figure 17: Prototype in Neutral Configuration

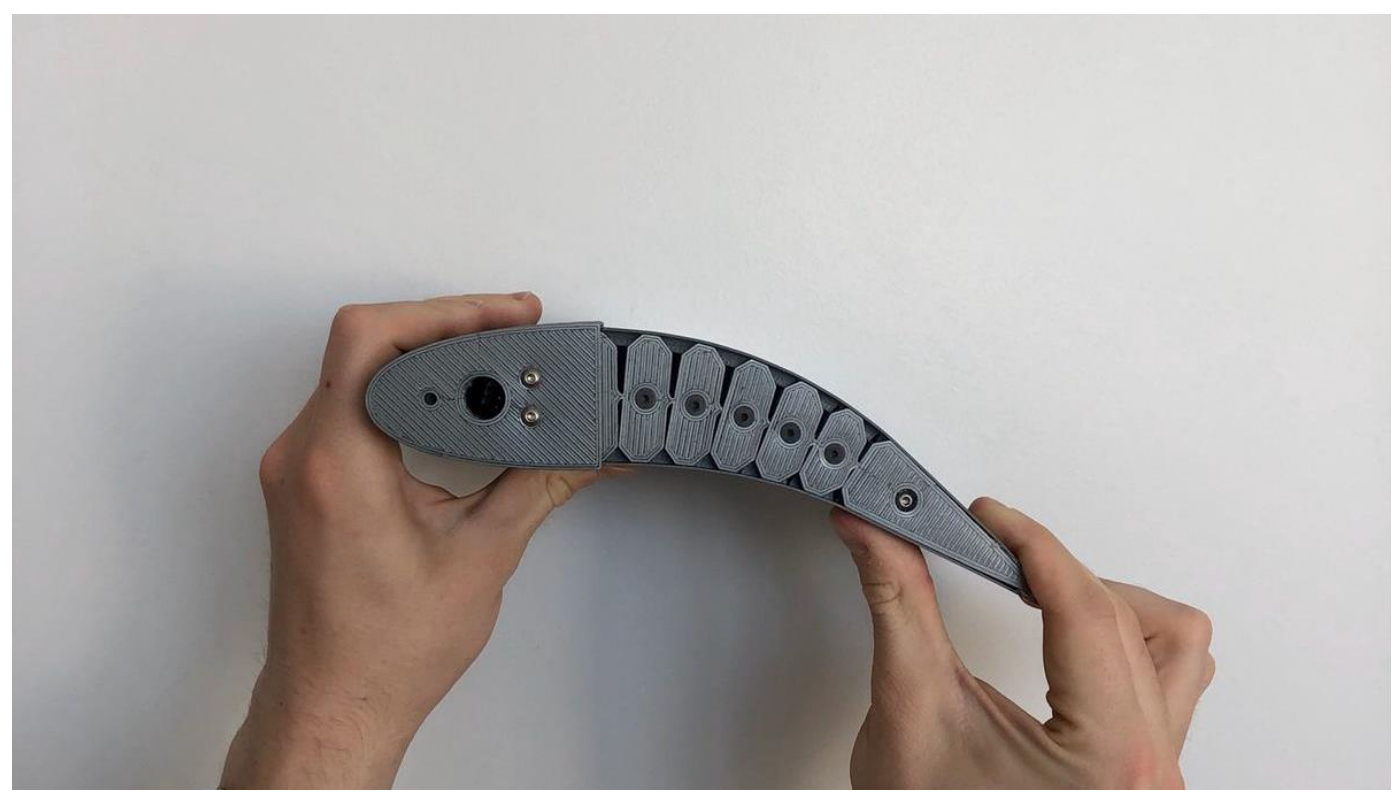

Figure 18: Prototype in Maximum Deflection Configuration 


\subsection{Analysis of Selected Design}

\subsection{Finite Element Analysis}

The final rib design was used for finite element analysis within SolidWorks to model how the rib would deform as required during different flight conditions. For simplicity, the affect of aerodynamic load on the cross-sectional shape was ignored. Additionally, by creating the deformed models using finite element analysis, they could then be exported and modeled with the skin panels attached for CFD analysis to be conducted.

For simplicity of the finite element analysis, the material was treated as fully solid and homogeneous. This would not be directly indicative of the behaviour of the manufactured prototype as it was produced on a 3-D printer with layers, perimeters, and infill. The chosen material for the finite element analysis was ABS plastic, since it is one of the most common 3-D printing materials. The required material properties were pre-populated in SolidWorks as follows: $E=2 G P a, v=0.394$, and $T S=30 \mathrm{MPa}$.

For the finite element analysis, constraints were made to ensure accuracy of the results. The rigid leading-edge portion of the wing rib had a fixed geometry constraint applied, meaning that it had zero DOF and would not deform under load. A restriction on self-penetration was applied on the surfaces of the elements in the morphing section. This meant that when a flexure had displaced to the point of causing contact between elements, the sections would collide and distribute the load, instead of allowing the part to pass through itself. Finally, the actuation load was applied as a fixed vertical load on the rigid trailing edge portion of the rib. The force was set strictly vertical and normal to the surface because as the rib translated and rotated, the normal force would become a follower force to the surface on which it was acting. This would make the system non-linear and lead to a more complex analysis being required. When SolidWorks detects that the FE analysis is leading to large displacement on a part/assembly it will automatically switch to a large displacement solver to improve the accuracy of the results. Documentation provided by Dassault Systemes describes the way the normal solver works as follows, "The full load is applied at once. Source and target pairs are set based on the initial configuration and remain unchanged during contact iterations. Normals to contact areas are also based on the initial configuration and remain unchanged during contact. Inaccurate results or convergence difficulties might occur if these assumptions are not valid." [20]. A description of how the large displacement solver is also provided as follows, "Loads are applied gradually and uniformly in a number of steps up to their full values. The software sets the number of steps based on deformation results. Source and target pairs and normals to contact areas are evaluated at each solution step." [20].

The main objective of this finite element analysis was to confirm the general shape that would be achieved under deformation. By understanding how the rib will deform, it can be controlled to get the desired trailing edge deflection angle. These deflection angles will be used to compare the morphed airfoil with a hinged flap combination in the following section. The V4 model was tested in the finite element analysis with varying loads until deformed models were created with trailing edge deflection angles of 0 , 10, 20, and 30 degrees. The undeformed rib along with the respective increases in trailing edge deflections can be seen below. 


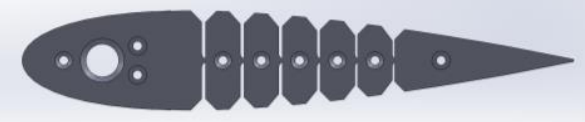

L.

Figure 19: FEA Model with Zero Degrees Trailing Edge Deflection

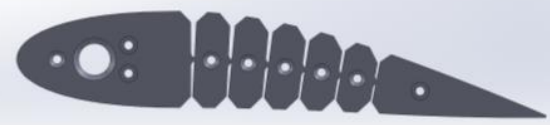

$L$

Figure 20: FEA Model with Ten Degrees Trailing Edge Deflection

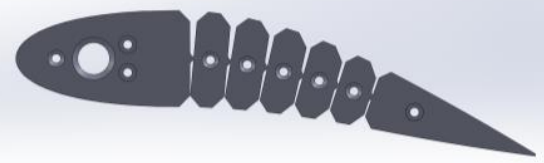

$\leftarrow$

Figure 21: FEA Model with Twenty Degrees Trailing Edge Deflection

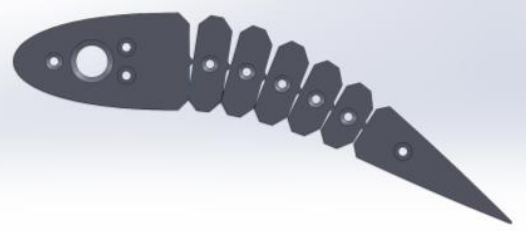

L.

Figure 22: FEA Model with Thirty Degrees Trailing Edge Deflection 


\subsection{Computational Fluid Dynamics}

The goal of this section was to conduct CFD analysis of the morphed wing design at $0,10,20$, and 30 degrees of trailing edge deflection. Analysis would also be done on a hinged flap design made up of the same base airfoil (NACA 0018), with the hinged flap making up 30\% of the chord length. The hinged flap design would also be tested at $0,10,20$, and 30 degrees of flap deflection. The data was collected, and the results were analyzed with conclusions drawn in the following sections. The computational fluid dynamics analysis conducted for this section was done so using ANSYS Fluent. To do the CFD analysis, the CAD models first had to be prepared in SolidWorks. The morphed rib models produced in the finite element analysis were exported as new bodies and had the rigid leading-edge skin panels as well as the compliant skin panels applied. The models were then ready to be exported for use in the CFD analysis. Images can be seen below of both the hinged flap and morphing wing models prepared for CFD analysis.

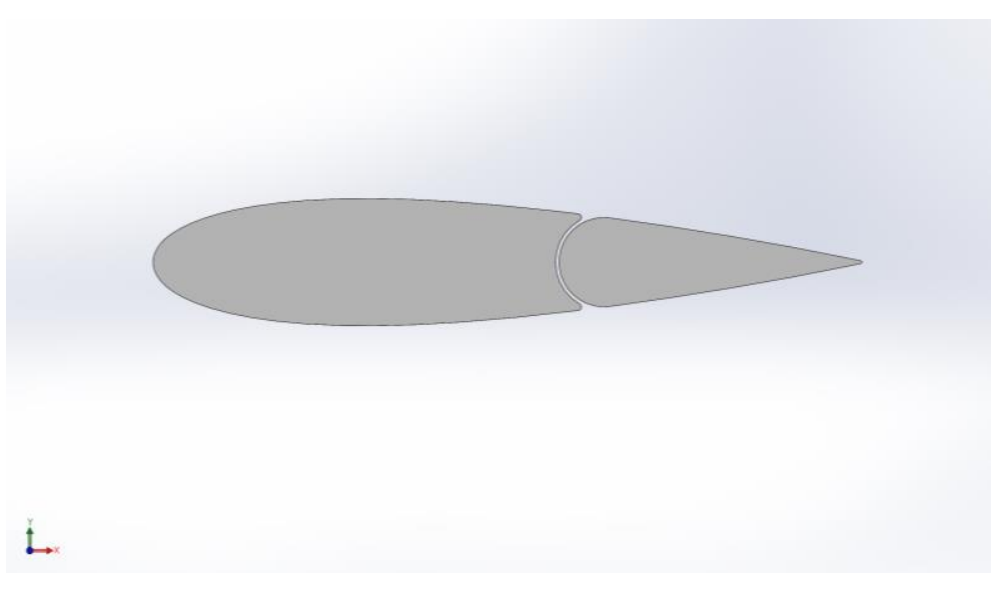

Figure 23: Hinged Flap CFD Model

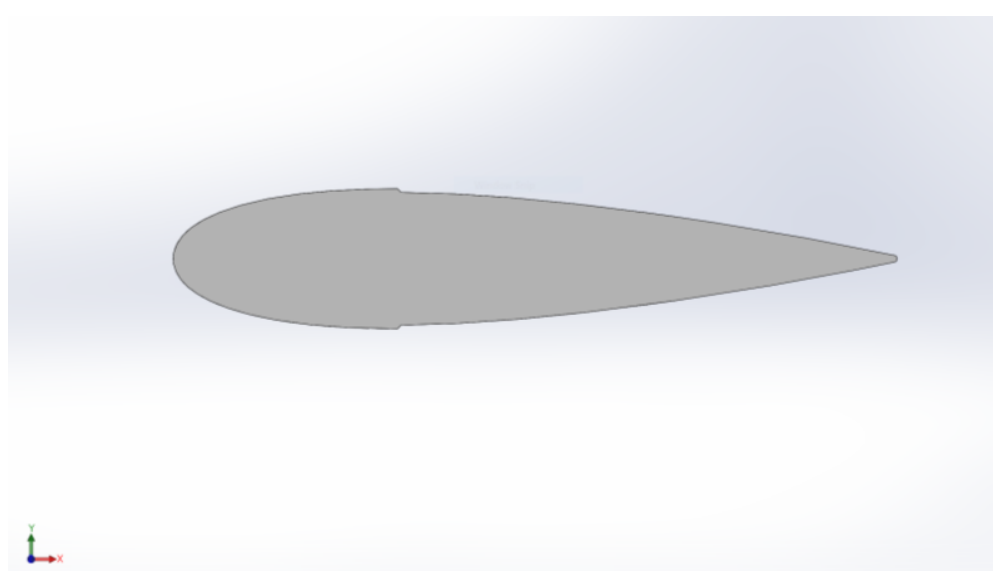

Figure 24: Morphing Wing CFD Model 
The CFD done for this analysis was all conducted as 2-D tests as this allowed for faster results. Additionally, by doing 2-D analysis, the only factors influencing the results would be the cross-sectional shape differences of the two flap types. The models were imported into ANSYS Design Modeler and had a control area generated around them with a $0.5 \mathrm{~m}$ radius extending out from the centre of the airfoil towards the leading-edge and a $0.5 \mathrm{~m}$ length rectangular shape extending out towards the trailing edge. An image of this control area can be seen in the following figure.

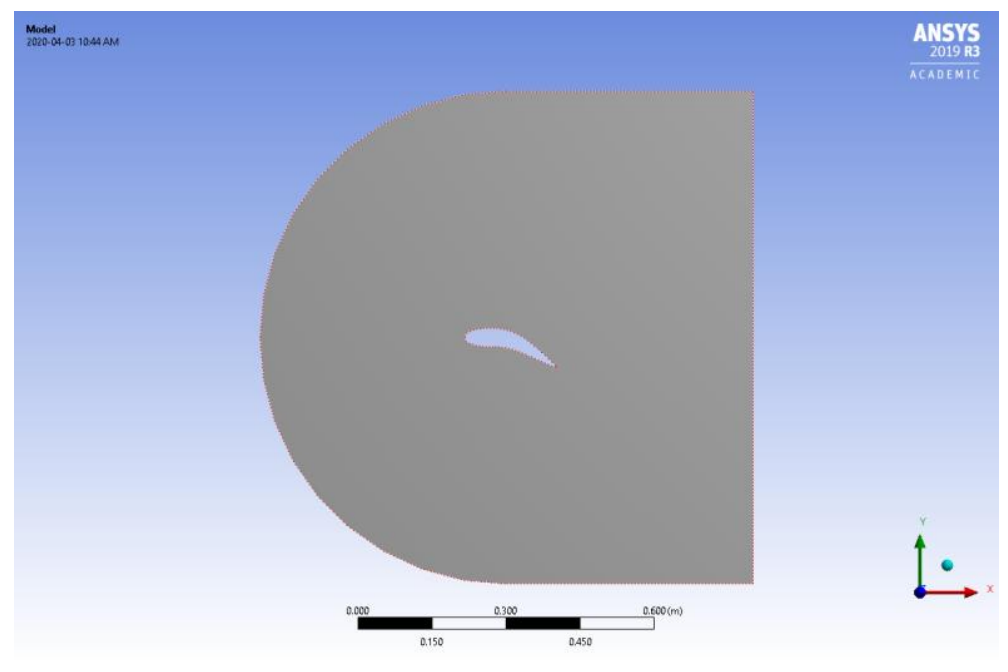

Figure 25: CFD Model Control Area

The models were then sent to be meshed for their analysis. For meshing, a quadratic element order was used. The maximum element size was set to $2.5 \mathrm{~mm}$, as this was found to provide allowable mesh element quality and mesh orthogonal quality values. The average mesh element quality from all tested models was above 0.98 , and the average mesh orthogonal quality was approximately 0.99 . The target skewness was left to its default value of 0.9. The growth rate of the element size was also left to its default value of 1.2. To improve the quality of the mesh, the smoothing was set to high. To improve the quality of the results while reducing unnecessary computation, a mesh refinement factor was set along the edges of the morphed wing and the main wing and hinged flap in their respective tests. The mesh refinement used was a factor of 3 . The mesh was then generated on the models. The final mesh statistics for all models had approximately 450,000 nodes and 150,000 elements. Images of the meshed thirty-degree morphed wing CFD model can be seen on the following page. Mesh sizing and quality was extremely important for the morphed wing model, especially around the step-down in the skin. It was assumed that this step-down would lead to adverse pressure gradients and become the source for most of the flow separation that would occur on this model. In the second image provided below, a more detailed view of the mesh can be seen. This image shows the refinement factor added around the morphed wing model to increase the accuracy of the modelling of the boundary layer and its resulting transition from laminar to turbulent. 


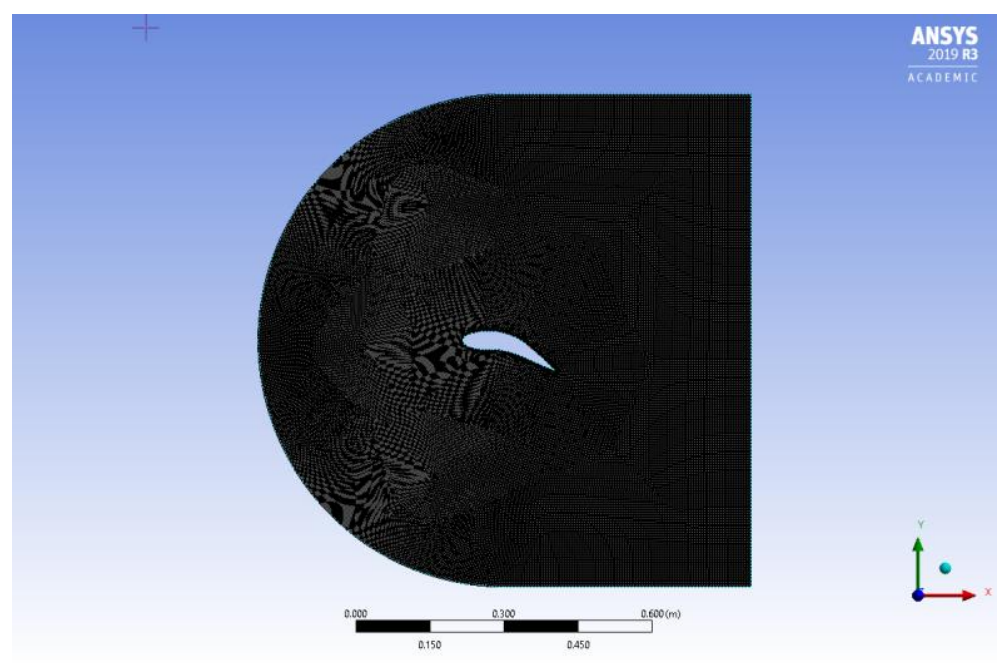

Figure 26: Meshed CFD Model

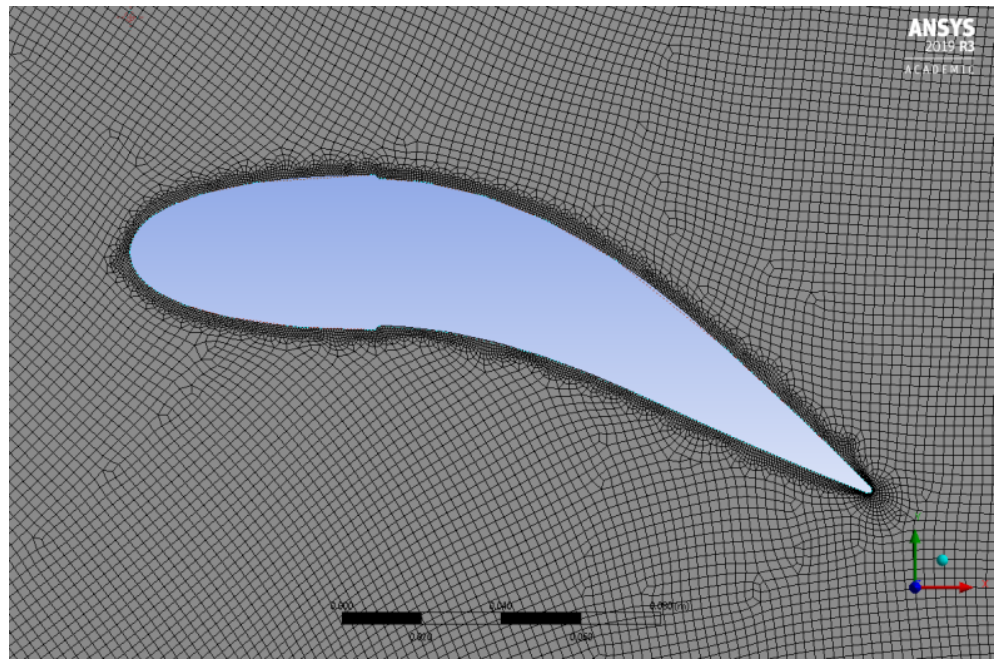

Figure 27: Detailed CFD Mesh View of Thirty Degree Morphed Wing

Following the meshing process, the models proceeded to Fluent where they had to undergo a final setup before the analysis could be run. The solver type used was a pressure-based solver, velocity formulation was absolute, time was set to steady-state, and 2-D space was set to planar. Under the setup section, the turbulence model was set to $\kappa-\omega$. The model was also set to use SST and a low Reynold's number correction. All the model constant values were left as the default values provided by Fluent. The fluid material used was air, with a constant density set at $1.225 \mathrm{~kg} / \mathrm{m}^{3}$ to represent sea-level air density. The viscosity of the fluid was also set to a constant value of $1.7894 \times 10^{-5} \frac{\mathrm{kg}}{\mathrm{m} * \mathrm{~s}}$. For the boundary conditions, the wing model was set to be a stationary wall. The upper and lower boundary of the control area were set to be moving walls, which would match the magnitude and direction of the incoming air velocity. The left side was set to be an inlet velocity and was changed for each set of tests. The tested inlet velocities were $20,40,60,80$, and $100 \mathrm{~m} / \mathrm{s}$. In each case the velocity had only an $\mathrm{x}$-component, meaning the airfoil was treated in each test as having a zero-degree AOA with respect to the undeformed shape or 
undeployed flap. The turbulence specification model of the inlet velocity was set to intensity and viscosity ratio. The turbulence intensity default value was $5 \%$ and the turbulent viscosity ratio was 10 . Under the reference values tab, the chord was set to 0.2 meters and the wing was given a unit span of 1 meter. Therefore, the reference wing area was $0.2 \mathrm{~m}^{2}$. Additionally, for ambient temperature STP conditions were used, so the value set for the analysis was $288.16 \mathrm{~K}$. To improve the accuracy of the results, all spatial discretization values under the solution methods were set to second-order upwind. Another step taken to improve the accuracy of the results was to reduce the threshold values for the residuals. All residuals were set to $1.0 \times 10^{-4}$. The solution initialization method was set to standard, computed from the inlet, and the reference frame was set to be relative to the cell zone. Finally, the number of iterations was set to 1000 and the simulation was run.

\subsection{Results of CFD Analysis}

At the completion of the CFD tests, results were found for the lift and drag of the hinged flap and morphed flap at deflection angles of 0,10, 20, and 30 degrees at 20,40,60,80 and $100 \mathrm{~m} / \mathrm{s}$. Traditionally on an aircraft flaps are used in the takeoff, climb, descent, and landing segments of flight. All these cases require the use of high-lift devices and the overall goal in these situations is to maximize the lift available to shorten the required field length for the aircraft as it will allow the aircraft to takeoff and approach the runway at a slower speed. Therefore, as an objective by which to gauge the results of the CFD analysis, the amount of lift generated will be a critical factor. Another factor to be considered in the CFD results is the lift-to-drag ratio. This term will represent how efficient the wing is at generating lift. This value is not as important for the descent and landing but is important for the takeoff and climb segments. It is important for these segments because for takeoff and climb because as the efficiency of the wing increases, the thrust required will decrease and as a result will decrease the overall mass fuel required for the mission. This reduction in required fuel weight then has an effect of reducing the amount of lift required in all stages of flight.

An average estimation for fuel used for the takeoff + climb and descent + landing segments is approximately $4.5 \%$ and $0.5 \%$ respectively of the total fuel weight stored on the aircraft [21]. Therefore, there is ample room for fuel savings due to increases in wing efficiency for the takeoff and climb segments.

The charts that follow breakdown the results found from the simulations. The first chart outlines the impact on the type of flap, its deflection angle, and its freestream airspeed on the resulting lift force that it generates. The second chart does the same but shows the generated drag force. The third chart shows how the type of flap, deflection angle, and freestream airspeed affect its lift-to-drag ratio. Finally, the last chart shows how the type of flap and its deflection angle impact its coefficient of lift. The notation in the chart legend appears as follows, MF-10, HF-30. For the notation, MF and HF refer to hinged flap and morphing flap respectively with the corresponding number referring to the angle of deflection applied at the trailing edge. Additionally, a full breakdown of the results can be found in Appendix A. 


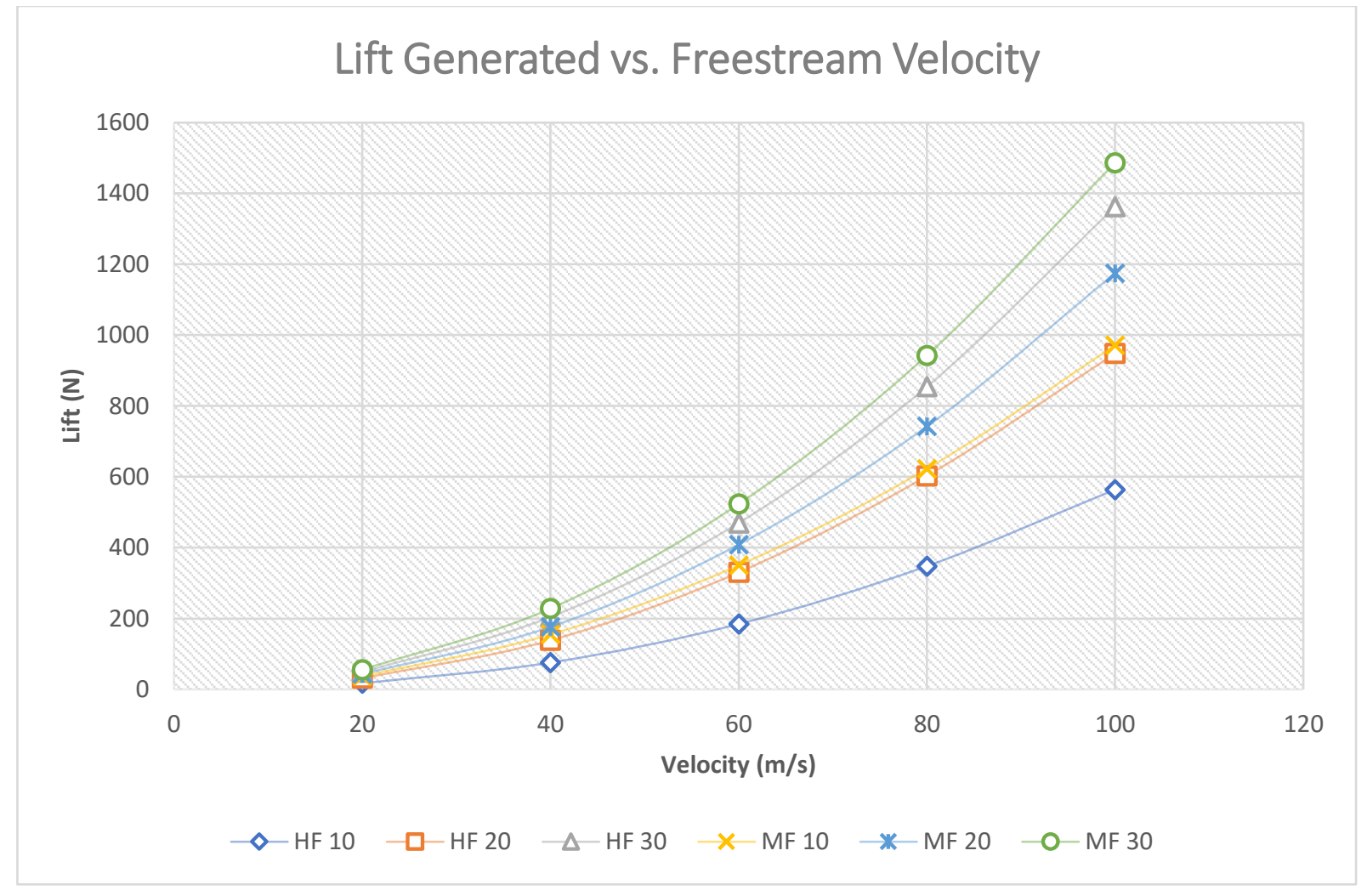

Figure 28: Lift vs. Velocity Comparison

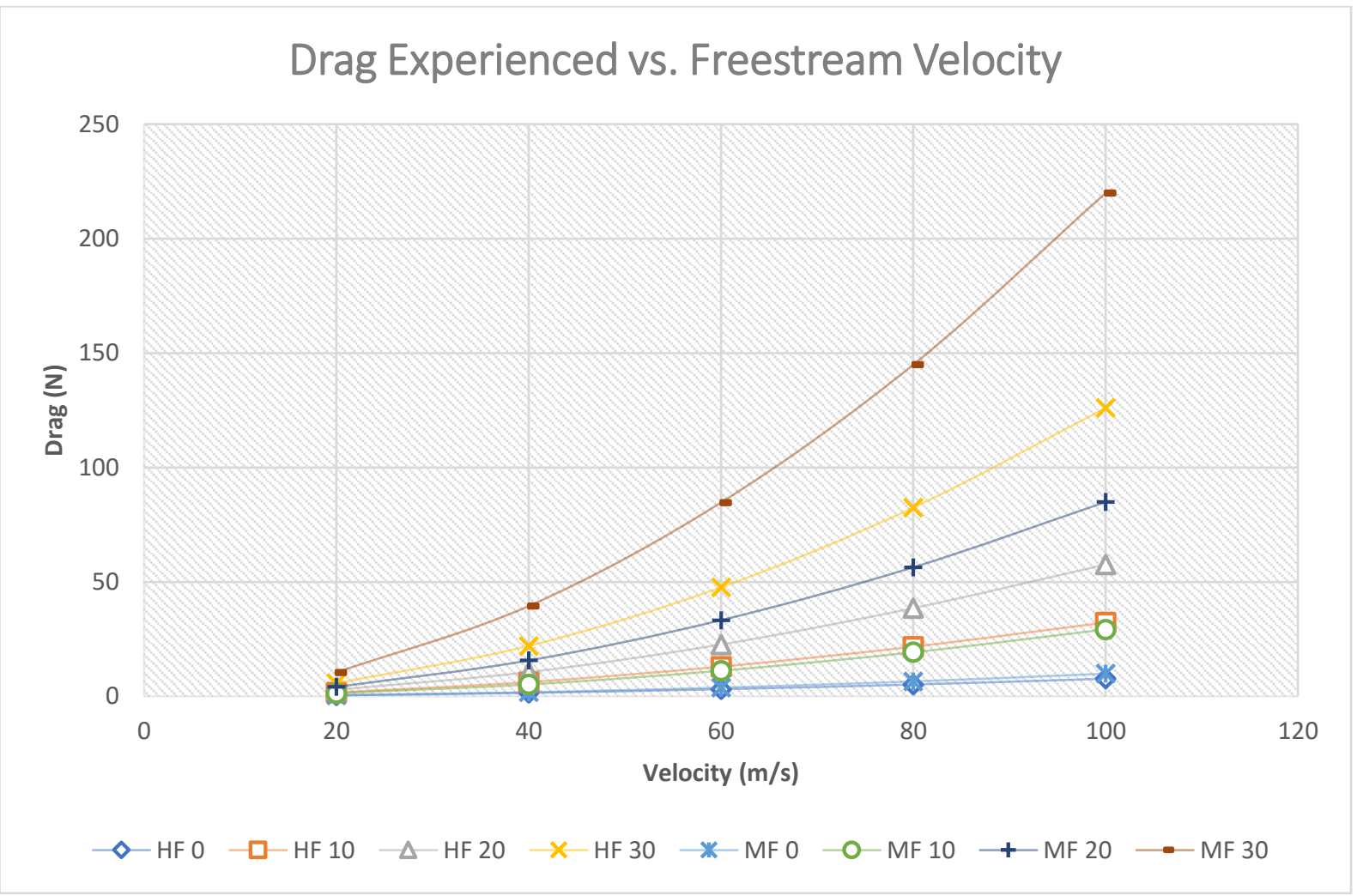

Figure 29: Drag vs. Velocity Comparison 


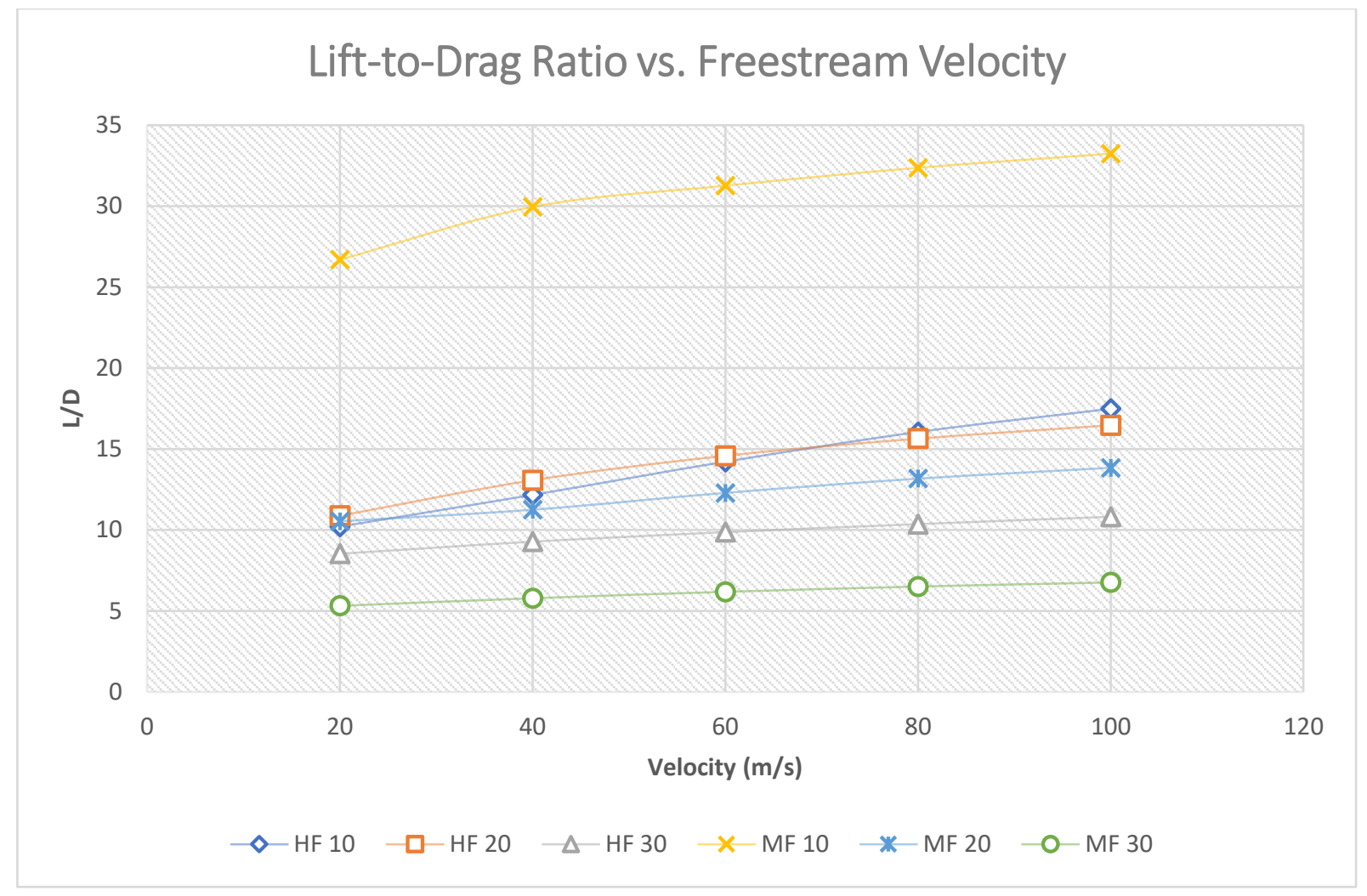

Figure 30: L/D vs. Velocity Comparison

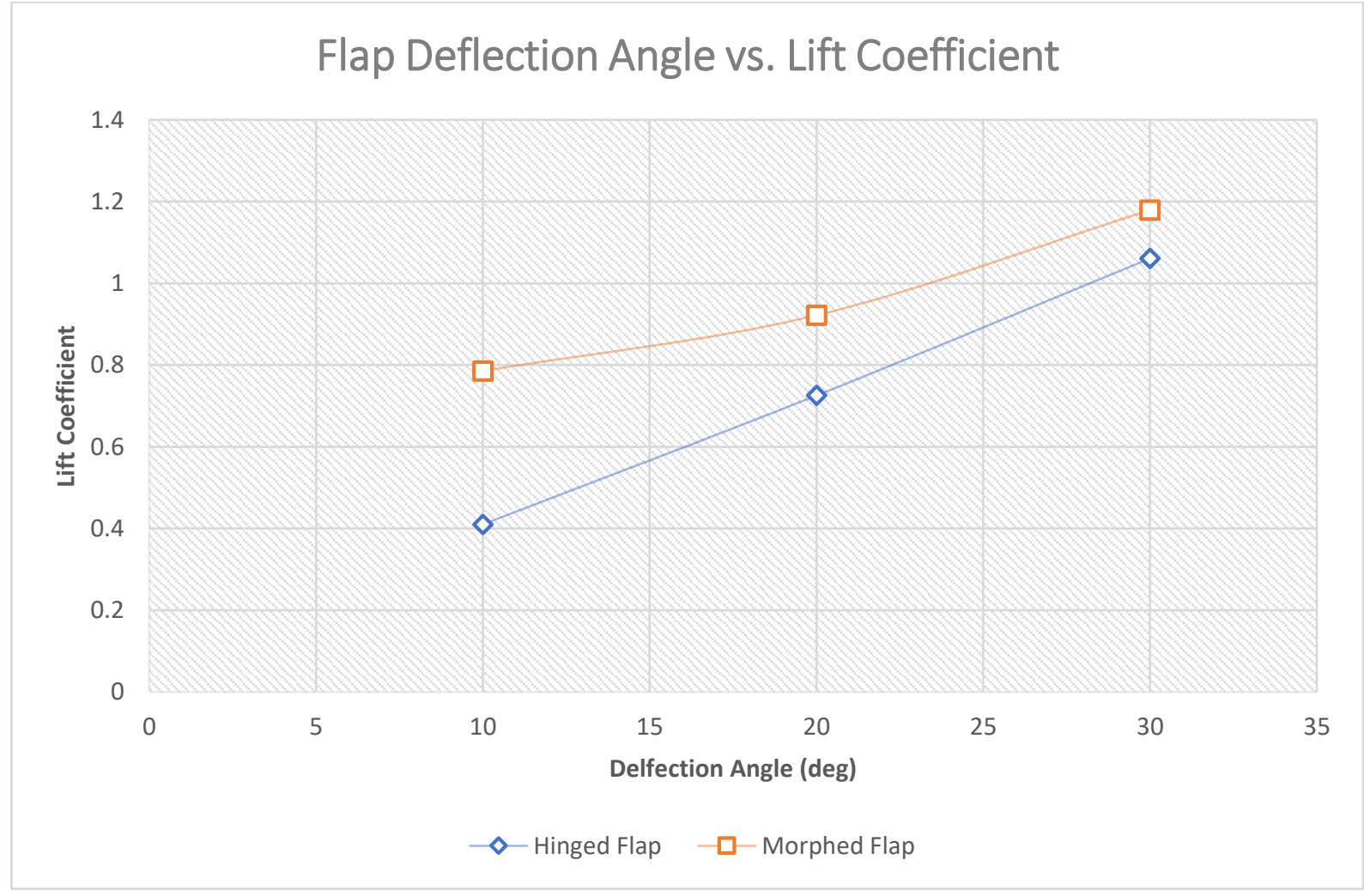

Figure 31: Deflection Angle vs. Lift Coefficient Comparison 
The results showed that for any given velocity, the morphing wing generated more light than the hinged flap. In the worst-case scenario at $30^{\circ}$ deflection and $100 \mathrm{~m} / \mathrm{s}$ airspeed, the morphing wing generates $9.12 \%$ more lift. In the best-case scenario at $10^{\circ}$ deflection and $20 \mathrm{~m} / \mathrm{s}$ airspeed, the morphing wing generates $115.04 \%$ more lift than the hinged flap design.

The results for drag differ from those of the lift results. In the $0^{\circ}$ deflection tests, the morphed wing saw up to $29 \%$ higher drag at high speed. However, when the $10^{\circ}$ tests were conducted, the morphing wing had drag values up to almost $18 \%$ lower in some tests. This trend reversed again for the 20 - and 30-degree tests. In both sets of tests, the drag of the morphing wing was greater and the rate of increase in the drag was also higher for the morphing wing.

The lift-to-drag chart gives a clear view of how the efficiency with which the wing generates lift as its airspeed and deflection angle are changed. In the best-case scenario, when the airspeed is $20 \mathrm{~m} / \mathrm{s}$ and the flap is deflected at $10^{\circ}$, the lift-to-drag ratio seen be the morphing wing is $161.41 \%$ higher than the hinged flap. As airspeed and deflection angle increases towards their maximum respective values tested, the hinged flap begins to perform slightly better. In the best-case scenario for the hinged flap at $30^{\circ}$ of deflection and $100 \mathrm{~m} / \mathrm{s}$ airspeed, the lift-to-drag ratio is $60.49 \%$ higher than the morphed wing.

The average lift coefficients calculated for the hinged flap at 10, 20, and 30 degrees of deflection were respectively; $0.410,0.725$, and 1.061 . The same values for the morphed wing were; $0.786,0.922$, and 1.179. This meant the lift coefficient was always higher for the morphed wing and in the case of the lift coefficient for the $10^{\circ}$ morphed wing, its lift coefficient was even higher than that of the $20^{\circ}$ hinged flap. On average across all three tested deflection angles, the lift coefficient for the morphed wing was approximately $43 \%$ higher than the hinged flap configuration.

The full list of lift, drag, and lift-to-drag ratio values found through the CFD analysis can be found in Appendix A. Additionally, reference images of all the pressure and velocity plots generated from the 100 $\mathrm{m} / \mathrm{s}$ airspeed tests can be found in Appendix B. 


\subsection{Conclusions}

This report served as an exploratory look into new methods and ideas by which a morphing wing could be designed. The goal of these designs was to increase the lift and the efficiency of the wing. These designs also carried a secondary goal of being simple. The purpose of this goal was to serve as a feasibility check so that any factors such as weight, part count, manufacturing, or maintenance would not singlehandedly act as the Achilles heel of the design.

The selected final design met most of these criteria under certain conditions. The use of compliant mechanisms was specifically chosen as they allow for replacing large traditional multi-link mechanisms with a single part. Additionally, compliant mechanisms have no backlash and do not require lubrication. Provided the high-stress flexural points are sufficiently designed, fatigue failure can be avoided and minimal to no maintenance will ever be required. At the time of writing, most advanced applications of compliant mechanisms, such as the 2-DOF gas thruster mount designed by Brighman Young University in partnership with NASA is designed to be 3-D printed out of titanium. This would be the easiest method of manufacturing; however, it would be prohibitively expensive. It is also expected with the vast reduction in the number of parts required, the overall weight of the system would decrease but further work would need to be done to verify this.

Initial designs and testing of the compliant mechanisms for this research used TPU, which was provided a very low barrier to entry of design as the base material is very flexible and compliant. This material however was not a feasible choice and did not reflect the properties of the materials a more detailed prototype would be constructed of. As a result, the focus shifted to more rigid and brittle materials that could be easily produced on a desktop 3-D printer. The brittle materials still proved relatively simple in the design of the flexures after initial testing on flexure widths and their allowable rotation angle before failure. The final design which featured six flexural points of $1.2 \mathrm{~mm}$ each that allowing five degrees of rotation (for a total of thirty degrees of trailing edge deflection) proved to be very robust and after $>1000$ maximum $\pm 30^{\circ}$ manual deflections showed no signs of fatigue or breakage. Therefore, these designs proved to be relatively easy to design and very reliable even when produced in rigid and brittle materials.

The skin served as an exploratory look into the design of a feasible skin panel that could easily move and morph with the underlying structures. From initial research it was found that most concepts and prototypes feature an elastomer skin, preferably an anisotropic material that could easily stretch in the chordwise direction yet remain stiff in the spanwise direction. The tests done for this research involving elastomer skin materials yielded negative results. Application and fastening the skin proved to be difficult and inconsistent. The elastomer skin even when pre-stretched before application still buckled when undergoing compression and showed signs of fatigue after minimal testing. The design converged on a solution that incorporated a thin skin panel made from the same material as the rib structures. The skin panel was thin enough that it could easily bend, but the rib was designed so that the skin panel would never have to change in length when the surface of the wing underwent compression or extension. This meant a material such as the PETG skin panel that was made was fully suitable for the application. In order to attach the skin panel but still have it move with the changing wing camber required the skin panel to be fixed at one end, with the rest being allowed to slide within a channel in the rib structure. This proved to be a very effective design, as the assembly was much easier and repeatable when compared to the elastomer versions. The added flexural points to the skin panel proved to be paramount to the success of the skin panel design as previous versions resulted in excessive longitudinal stiffness of the panels. This stiffness resulted in 
excessive friction and actuation force within the ribs, which commonly resulted in the failure of the system. The placement of these flexures also proved to be very important as they needed to allow for bending between the rigid elements of the rib but not bind while translating between then elements during morphing.

Once the major design and manufacturing tasks were completed the main analysis done to test the viability of the design was a computational fluid dynamics analysis conducted in ANSYS Fluent. The models were prepared in SolidWorks Large Displacement FEA solver to get the morphed wing shape. In all tests conducted in the CFD analysis, the morphing wing concept produced a higher lift, with the best results yielding a $115 \%$ increase in lift over the hinged flap design. Additionally, for the $10^{\circ}$ deflection tests the lift-to-drag ratio was $161 \%$ higher for the morphing wing. This changed as the deflection angle increased to 20 and 30 degrees, when the hinged flap had higher lift-to-drag ratios. This was due to a combination of the higher effective angle of attack of the morphing wing and its step-down in the skin which caused visible flow separation much earlier along the chord of the wing when compared to the hinged flap. The morphed flap at $10^{\circ}$ of deflection was the best performer of all configurations tested. It had the highest lift-to-drag ratio as it was nearly double that of the next best performer. Additionally, this design generated more lift than the hinged flap when deflected to $20^{\circ}$. Therefore, for situations such as takeoff and climb where the flap deflection is typically between $5-15^{\circ}$, there is a significant performance and efficiency gain that can be made with the morphing wing design. These efficiency gains in lift generation could represent significant fuel and costs savings for an airline. In higher deflection situations, the lift generated by the morphing wing is still higher but comes at the cost of increased drag over the hinged flap. Additionally, the earlier chordwise location of the flow separation leads to the assumption that the morphing wing will also stall at a lower deflection angle than the hinged flap. This is not ideal, but the topics discussed in the future work portion aim to solve these problems.

There are several ways that work can be continued on this project to increase the efficiency and feasibility of this design. One of the first ways in improving the feasibility is to develop a proper sliding mechanism to attach the skin to the rib structure. Research into lubrication and design of such a sliding mechanism would improve the fit and further reduce actuation forces on the wing. As discussed in the CFD results, the step-down in the skin lead to flow separation at higher deflection angles. The step size in the models made for this research project was based on factors such as layer resolution of the printers used in the manufacturing process and the scale at which the wing was produced. It is assumed that as the wing is scaled up to that of even a Cessna 172, the ratio of the step size to chord length would be drastically lower. This would be assumed to dramatically reduce the amount of flow separation; however, it needs to be tested further to validate. Furthermore, the flexures were all contained in the middle third of the wing and all had equal rotational displacements. This should be tested further in CFD to see how the placement and magnitude of rotational displacement at each flexure can reduce the amount of flow separation and improve efficiency at high deflection angles. Finally, manufacturing and similar procedures for FEA and CFD analysis should be conducted on the V5 design as it mitigates the step in the skin in favour of a translating split trailing edge.

The final design kept to the goal of improving the lift over a basic flap design and in the low deflection cases was dramatically more efficient. It even met the secondary goal of simplicity, with the rib itself only being comprised of only two pieces and showing no signs of fatigue or wear after all the testing. The skin design proved to be very effective as well with easy installation and the ability to still be a structurally integral part on its own, which neither could be said of the elastomer materials tested. With continued work on improving the flow separation, this design proves promising for even high-deflection cases. Overall the V4 rib design and the accompanying compliant skin panel design were very successful for their initial tests. 


\section{References}

[1] "Inventing a Flying Machine: The Breakthrough Concept," Smithsonian Air and Space Museum, [Online]. Available: https://airandspace.si.edu/exhibitions/wright-

brothers/online/fly/1899/breakthrough.cfm. [Accessed 5 April 2020].

[2] C. Cutler, "How The 4 Types Of Aircraft Flaps Work," Bold Method, 31 May 2018. [Online]. Available: https://www.boldmethod.com/learn-to-fly/aircraft-systems/how-the-four-types-ofaircraft-flaps-work/. [Accessed 1 April 2020].

[3] P. Novaković, M. Hornak, M. J. Zachar and N. Joncic, "3D Digital Recording of Archaeological, Architectural and Artistic Heritage," University of Ljubljana, Ljubljana, 2017.

[4] Hankook Tire Canada, "Tire Design Innovation Project," 2018. [Online]. Available: https://m.hankooktire.com/ca-en/technology/design-project/design-innovation.html. [Accessed 7 July 2019].

[5] NASA, "What is MADCAT?," 3 April 2019. [Online]. Available: https://www.nasa.gov/feature/ames/madcat. [Accessed 7 July 2019].

[6] G. Norris, "Flexsys and Aviation Partners Display Morphing Wing," Aviation Week Network, 4 November 2016. [Online]. Available: https://aviationweek.com/MorphingWing. [Accessed 18 July 2019].

[7] "Flexsys Morphing Airplane Wing," The Awesomer, 18 September 2017. [Online]. Available: https://theawesomer.com/flexsys-morphing-airplane-wing/445810/. [Accessed 18 July 2019].

[8] "Smart Airfoil," ETH Zurich, 2020. [Online]. Available: https://structures.ethz.ch/research/sa/smart-airfoil.html. [Accessed 1 April 2020].

[9] K. Loudon, A. Bouferrouk, B. Coleman, F. Hughes, B. Lewis, B. Parsons, A. Cole and Y. Yao, "Further Development of a Variable Camber Morphing Mechanism Using the Direct Control Airfoil Geometry Concept," International Symposium on Sustainable Aviation, pp. 1-4, July 2018.

[10] F. Gandhi and P. Anusonti-Inthra, "Skin Design Studies for Variable Camber Morphing," Smart Materials and Structures, vol. 17, no. 1, pp. 15-25, 2008.

[11] T. Takahashi, T. Yokozeki and Y. Hirano, "Development of a Variable Camber Wing with Morphing Leading and Trailing Sections Using Corrugated Structures," Journal of Intelligent Material Systems and Structures, vol. 27, no. 20, pp. 2827-2836, 2016.

[12] O. S. Gabor, A. Koreanschi, R. M. Botez, M. Mamou and Y. Mebarki, "Numerical Simulation and Wind Tunnel Tests Investigation and Validation of a Morphing Wing-Tip Demonstrator Aerodynamic Performance," Aerospace Science and Technology, vol. 53, pp. 136-153, 2016. 
[13] C. Haubursin, "The man-made world is horribly designed. But copying nature helps," Vox Media, 2017. [Online]. Available: https://www.vox.com/videos/2017/11/9/16628106/biomimicrydesign-nature. [Accessed 27 March 2020].

[14] "How The Bullet Trains Found Their Efficient Design," Scrap Labs, 18 February 2018. [Online]. Available: http://blog.scraplabs.in/scraplabs-stories/biomimicry. [Accessed 1 April 2020].

[15] L. L. Howell, S. P. Magleby and B. M. Olsen, Handbook of compliant mechanisms, Chichester, UK: Wiley, 2013.

[16] "Overview of materials for Polylactic Acid (PLA) Bipolymer," MatWeb, [Online]. Available: http://www.matweb.com/search/DataSheet.aspx?MatGUID=ab96a4c0655c4018a8785ac4031b9 278\&ckck=1. [Accessed 20 February 2020].

[17] "Overview of materials for PETG Copolyester," MatWeb, [Online]. Available: http://www.matweb.com/search/datasheet_print.aspx?matguid=4de1c85bb946406a86c52b688 e3810d0. [Accessed 20 February 2020].

[18] "Overview of materials for Thermoplastic Polyurethane, Elastomer, Glass Filled," MatWeb, [Online]. Available:

http://www.matweb.com/search/datasheettext.aspx?matguid=2fe782a31c4b4bed984b4965176 2b086. [Accessed 20 February 2020].

[19] "Wonderful Ocean Fish Grand View Garden," Baike, 11 July 2011. [Online]. Available: http://tupian.baike.com/87338/8.html?prd=zutu_thumbs. [Accessed 1 April 2020].

[20] "Large Displacement Solution," Dassault Systemes, 2019. [Online]. Available: http://help.solidworks.com/2019/english/SolidWorks/cworks/c_Large_Displacement_Solution.ht m. [Accessed 1 April 2020].

[21] D. Raymer, "Aircraft Design: A Conceptual Approach 5th Edition," Washington D.C., AMER Institute od Aerona, 1992, p. 12. 


\section{Appendix A: Full CFD Results Data}

Table 2: Full CFD Results Data

\begin{tabular}{|c|c|c|c|c|c|c|c|c|c|}
\hline \multirow{4}{*}{$\begin{array}{c}\text { Velocity } \\
(\mathrm{m} / \mathrm{s})\end{array}$} & \multirow{4}{*}{$\begin{array}{c}\text { Values } \\
(\mathbf{N}, \mathbf{N}, \\
\mathbf{n} / \mathbf{a})\end{array}$} & \multicolumn{8}{|c|}{ Flap Type } \\
\hline & & \multicolumn{4}{|c|}{ Hinged } & \multicolumn{4}{|c|}{ Morphed } \\
\hline & & \multicolumn{8}{|c|}{ Deflection Angle (deg) } \\
\hline & & $\mathbf{0}$ & 10 & 20 & 30 & 0 & 10 & 20 & 30 \\
\hline \multirow{3}{*}{20} & $\mathbf{L}$ & 0.307 & 17.224 & 31.277 & 48.875 & -0.034 & 37.038 & 43.060 & 55.438 \\
\hline & D & 0.441 & 1.687 & 2.875 & 5.728 & 0.477 & 1.387 & 4.086 & 10.426 \\
\hline & L/D & 0.698 & 10.213 & 10.879 & 8.533 & -0.071 & 26.698 & 10.538 & 5.317 \\
\hline \multirow{3}{*}{40} & $\mathbf{L}$ & 3.889 & 75.701 & 138.503 & 204.424 & -0.763 & 155.023 & 176.307 & 228.157 \\
\hline & D & 1.530 & 6.221 & 10.594 & 22.032 & 1.761 & 5.176 & 15.669 & 39.439 \\
\hline & L/D & 2.541 & 12.168 & 13.073 & 9.278 & -0.433 & 29.952 & 11.252 & 5.785 \\
\hline \multirow{3}{*}{60} & $\mathbf{L}$ & 9.368 & 184.481 & 330.242 & 469.798 & -1.913 & 350.270 & 408.819 & 523.287 \\
\hline & D & 3.121 & 12.977 & 22.646 & 47.616 & 3.786 & 11.204 & 33.257 & 84.657 \\
\hline & L/D & 3.002 & 14.216 & 14.583 & 9.866 & -0.505 & 31.262 & 12.293 & 6.181 \\
\hline \multirow{3}{*}{80} & $\mathbf{L}$ & -14.03 & 347.959 & 602.669 & 854.482 & -3.997 & 622.763 & 742.294 & 942.915 \\
\hline & D & 5.146 & 21.677 & 38.530 & 82.456 & 6.493 & 19.240 & 56.362 & 144.897 \\
\hline & L/D & -2.726 & 16.052 & 15.642 & 10.363 & -0.616 & 32.368 & 13.170 & 6.507 \\
\hline \multirow{3}{*}{100} & $\mathbf{L}$ & -22.91 & 563.962 & 948.134 & 1362.509 & -8.141 & 971.426 & 1174.919 & 1486.791 \\
\hline & D & 7.684 & 32.251 & 57.569 & 125.953 & 9.888 & 29.224 & 84.878 & 219.891 \\
\hline & L/D & -2.982 & 17.487 & 16.470 & 10.818 & -0.823 & 33.241 & 13.842 & 6.761 \\
\hline
\end{tabular}




\section{Appendix B: CFD Results Contour Plots}

All test images are taken from the $100 \mathrm{~m} / \mathrm{s}$ airspeed tests.

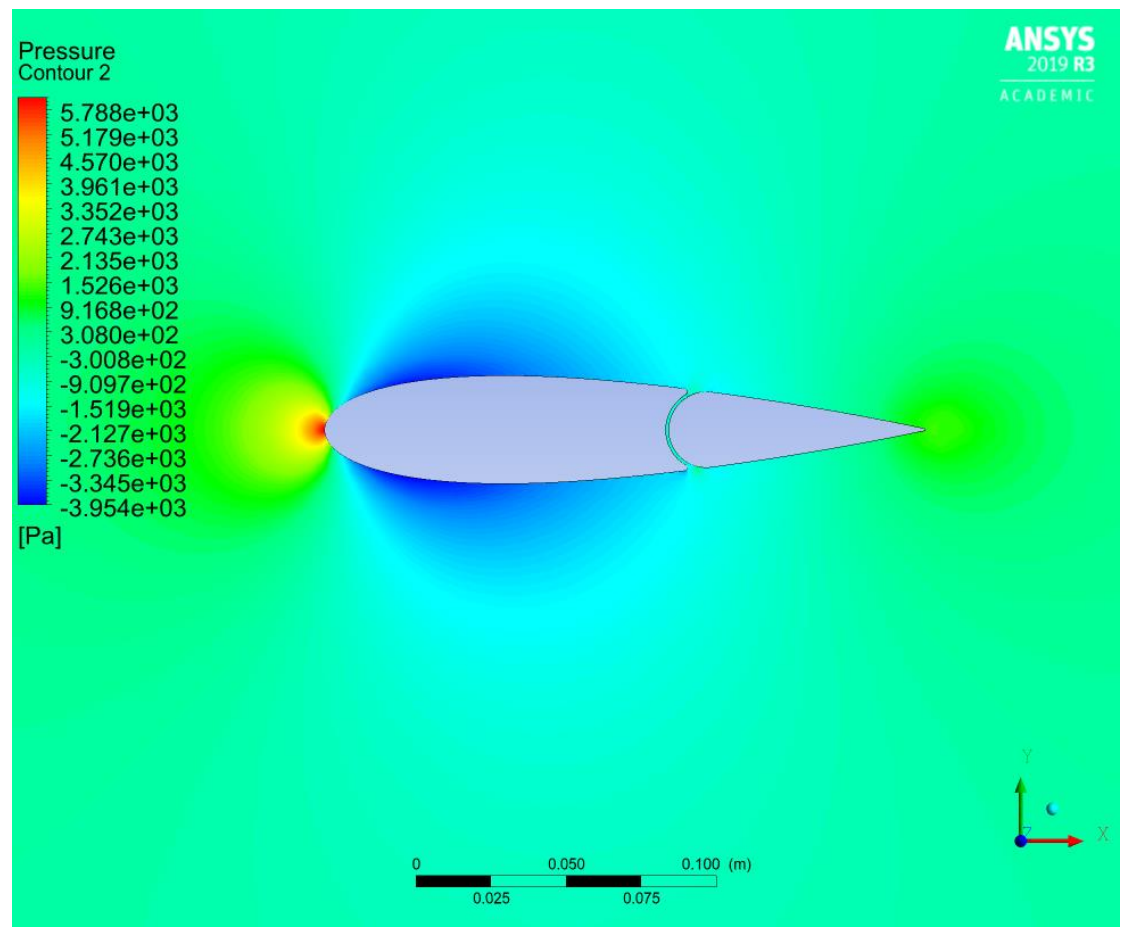

Figure 32: 0 deg Hinged Flap Pressure Contour

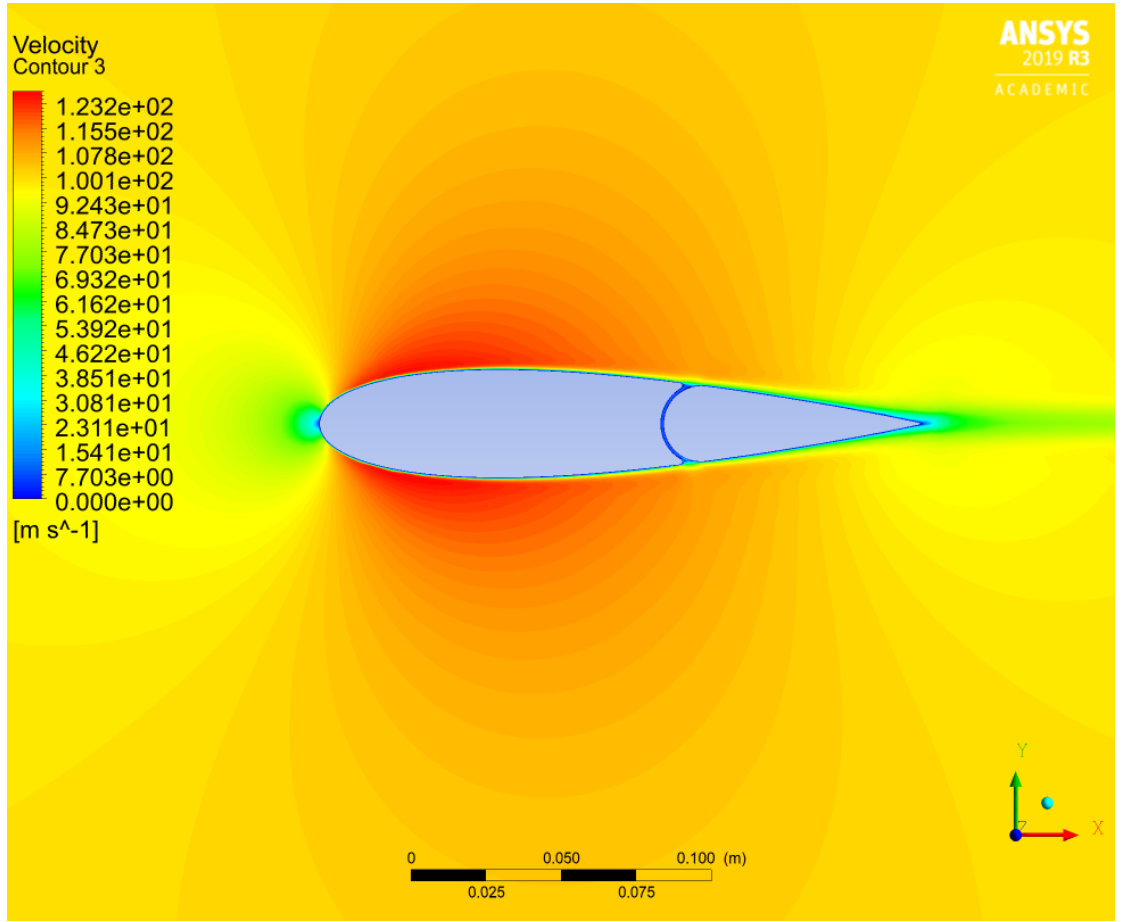

Figure 33: 0 deg Hinged Flap Velocity Contour 


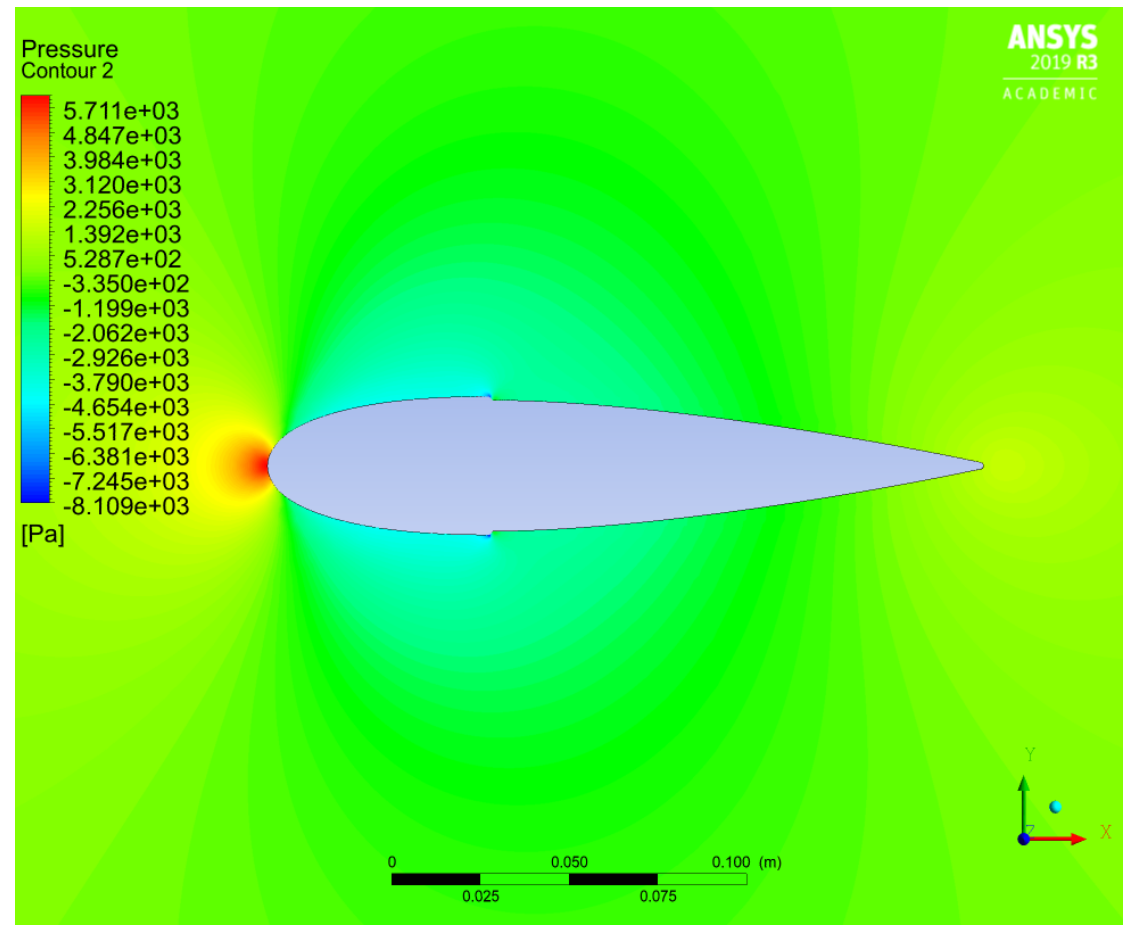

Figure 34: 0 deg Morphed Flap Pressure Contour

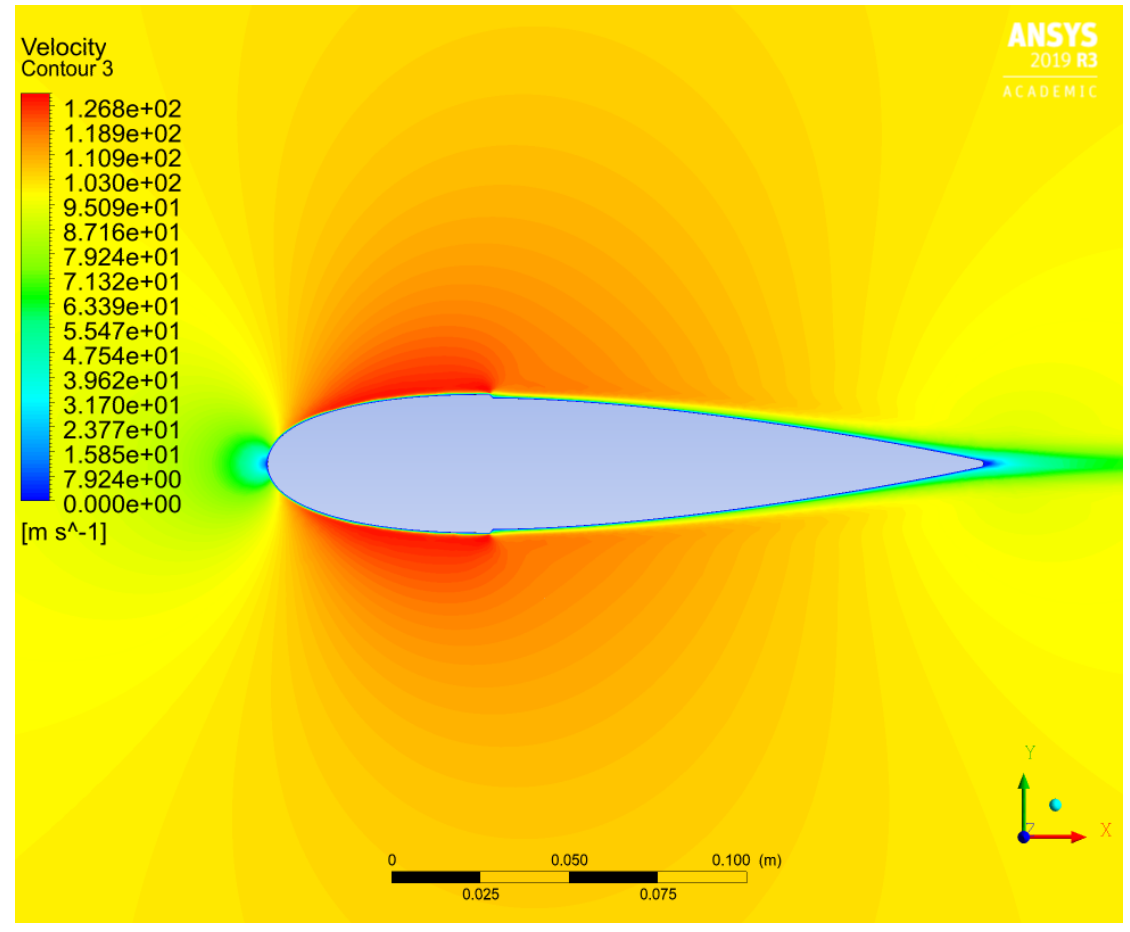

Figure 35: 0 deg Morphed Flap Velocity Contour 


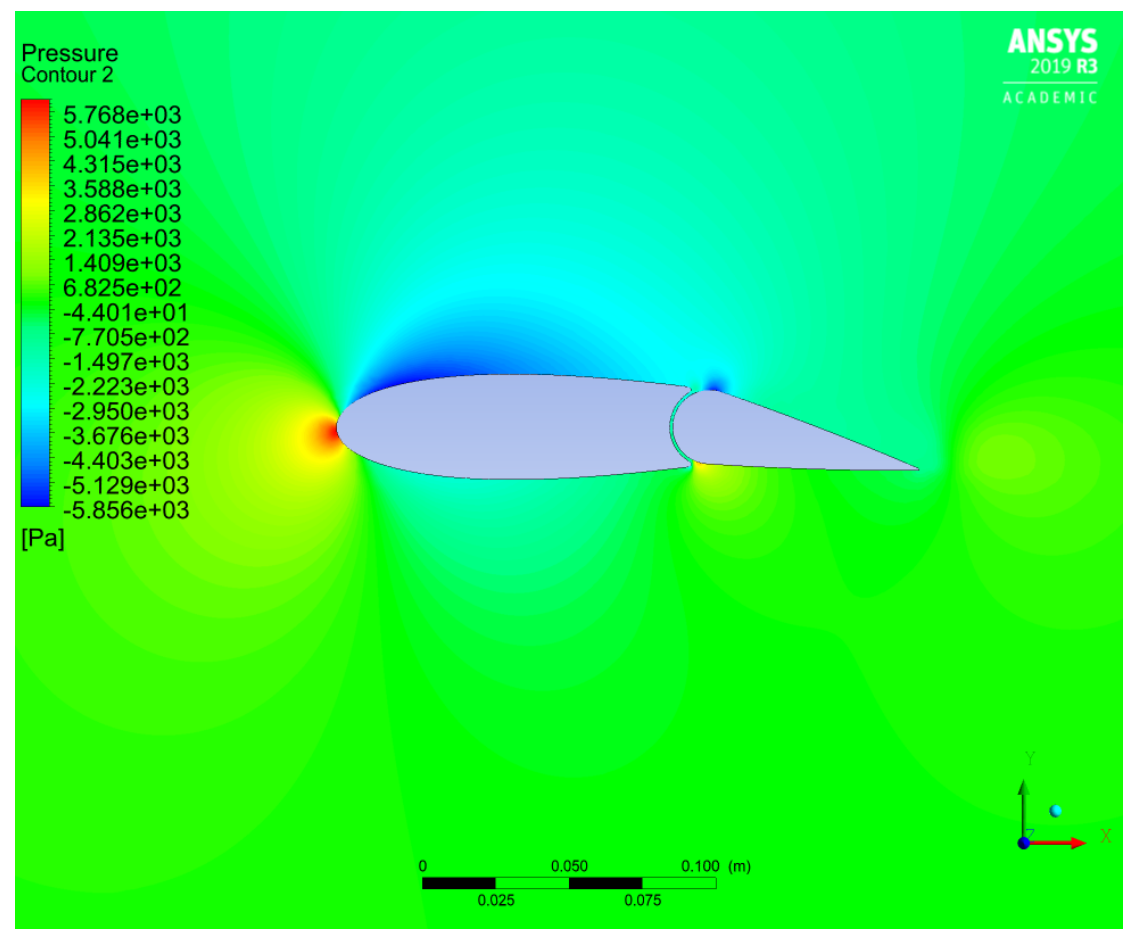

Figure 36: 10 deg Hinged Flap Pressure Contour

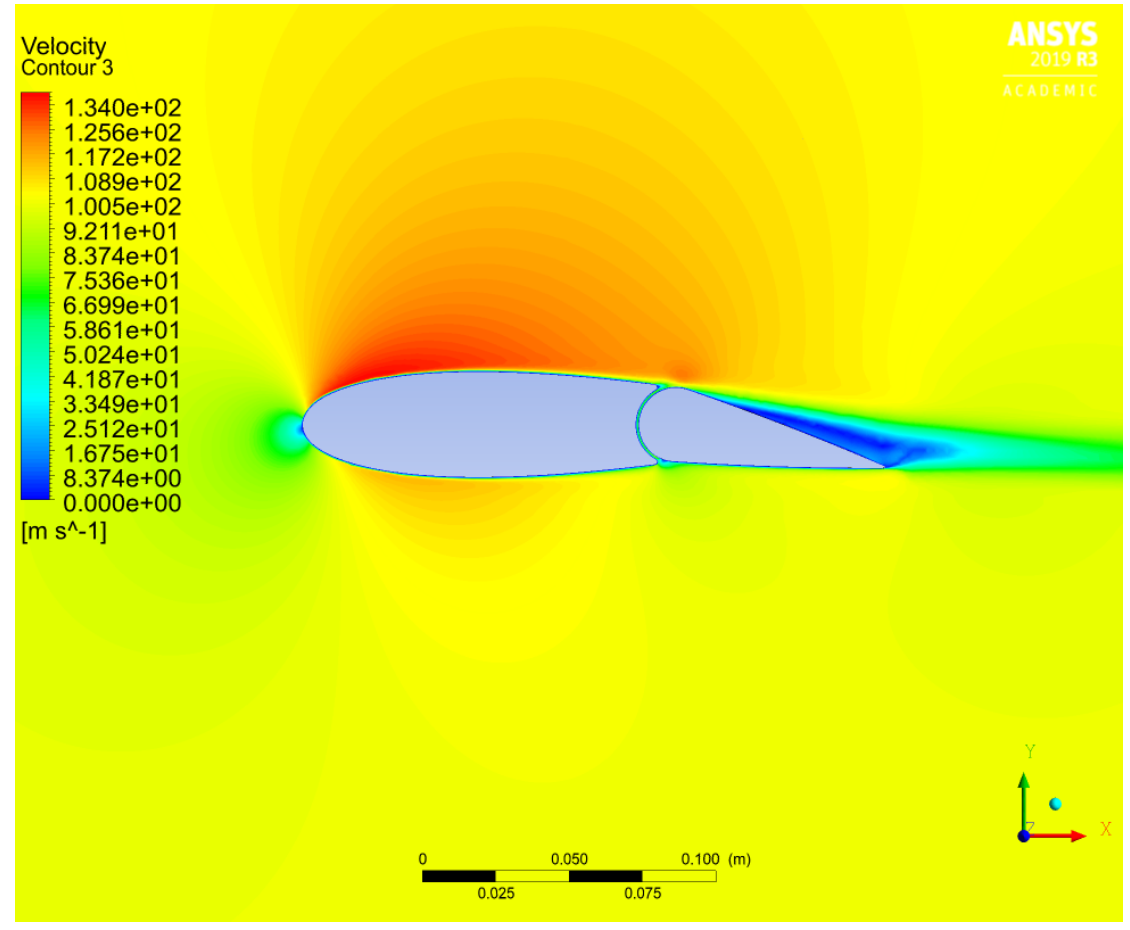

Figure 37: 10 deg Hinged Flap Velocity Contour 


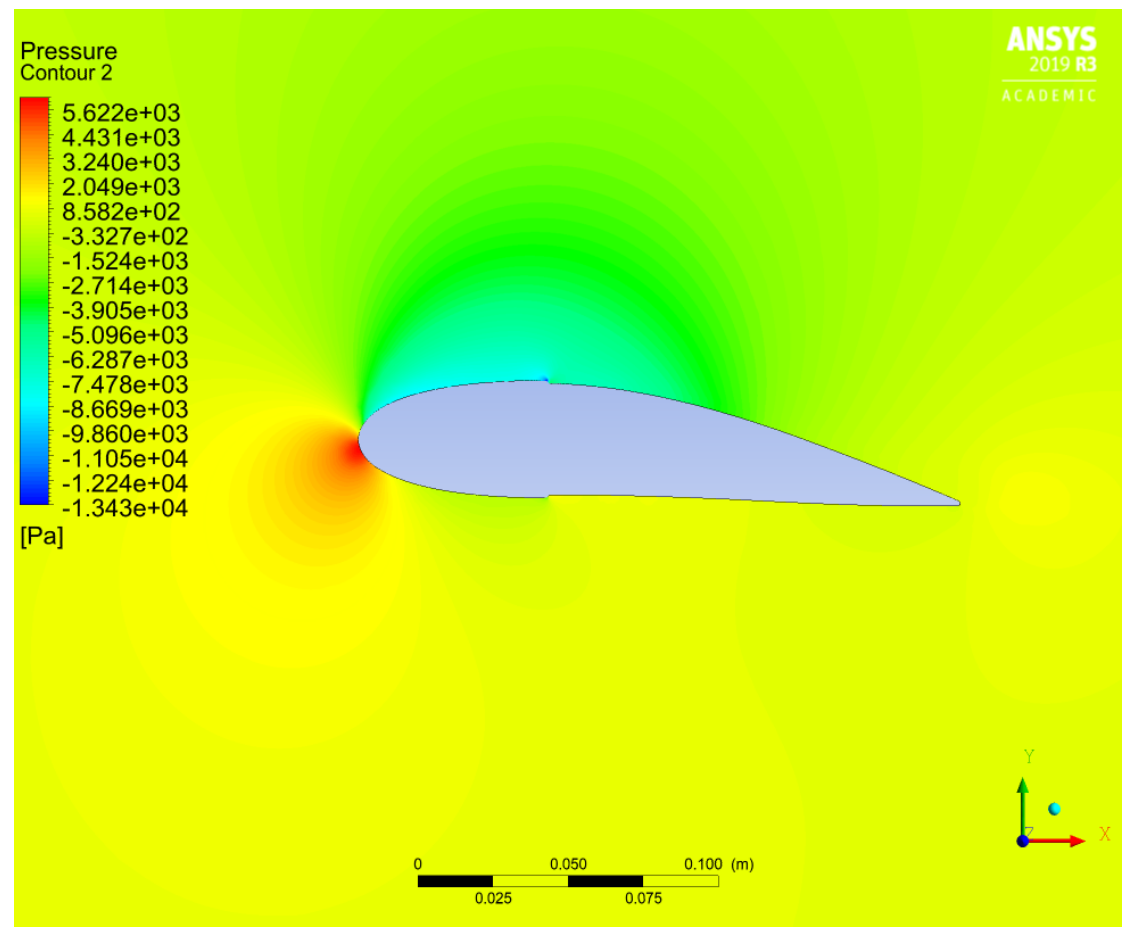

Figure 38: 10 deg Morphed Flap Pressure Contour

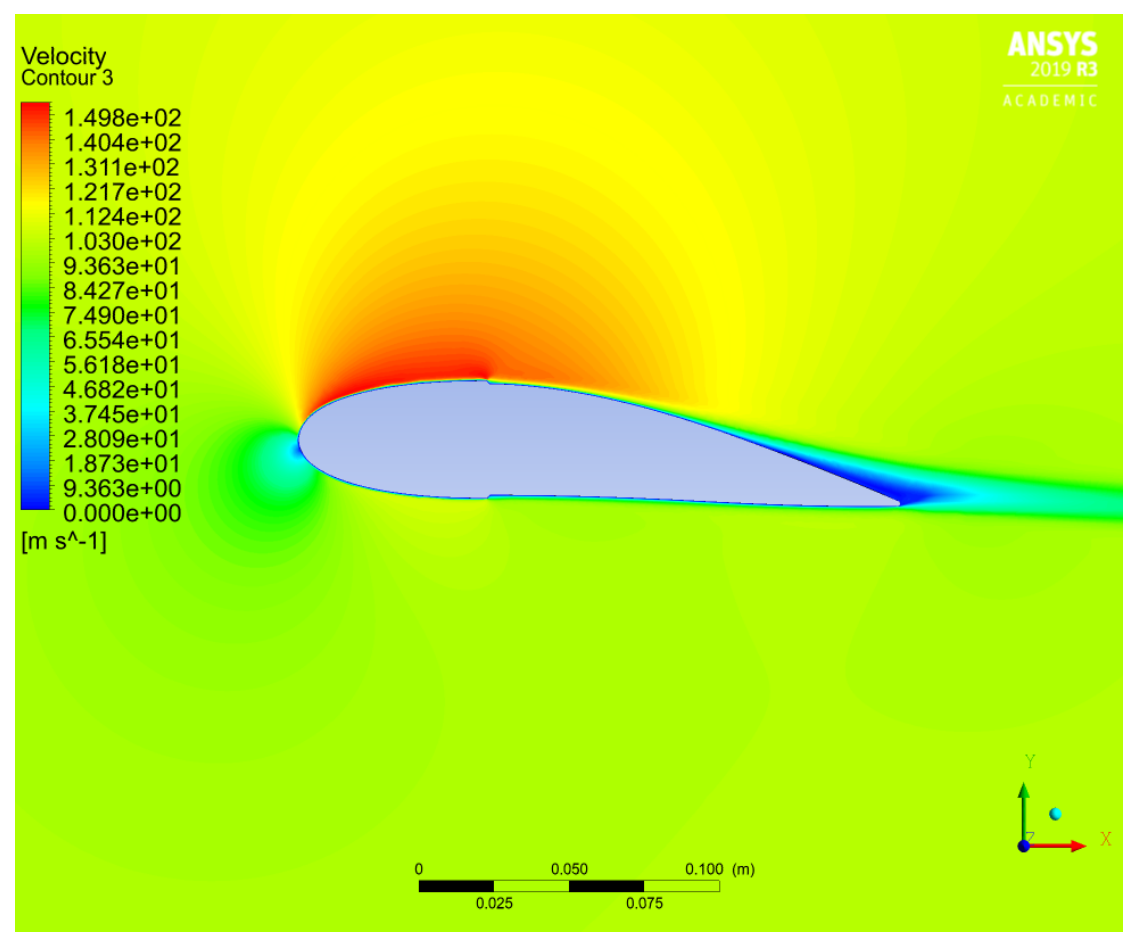

Figure 39: 10 deg Morphed Flap Velocity Contour 


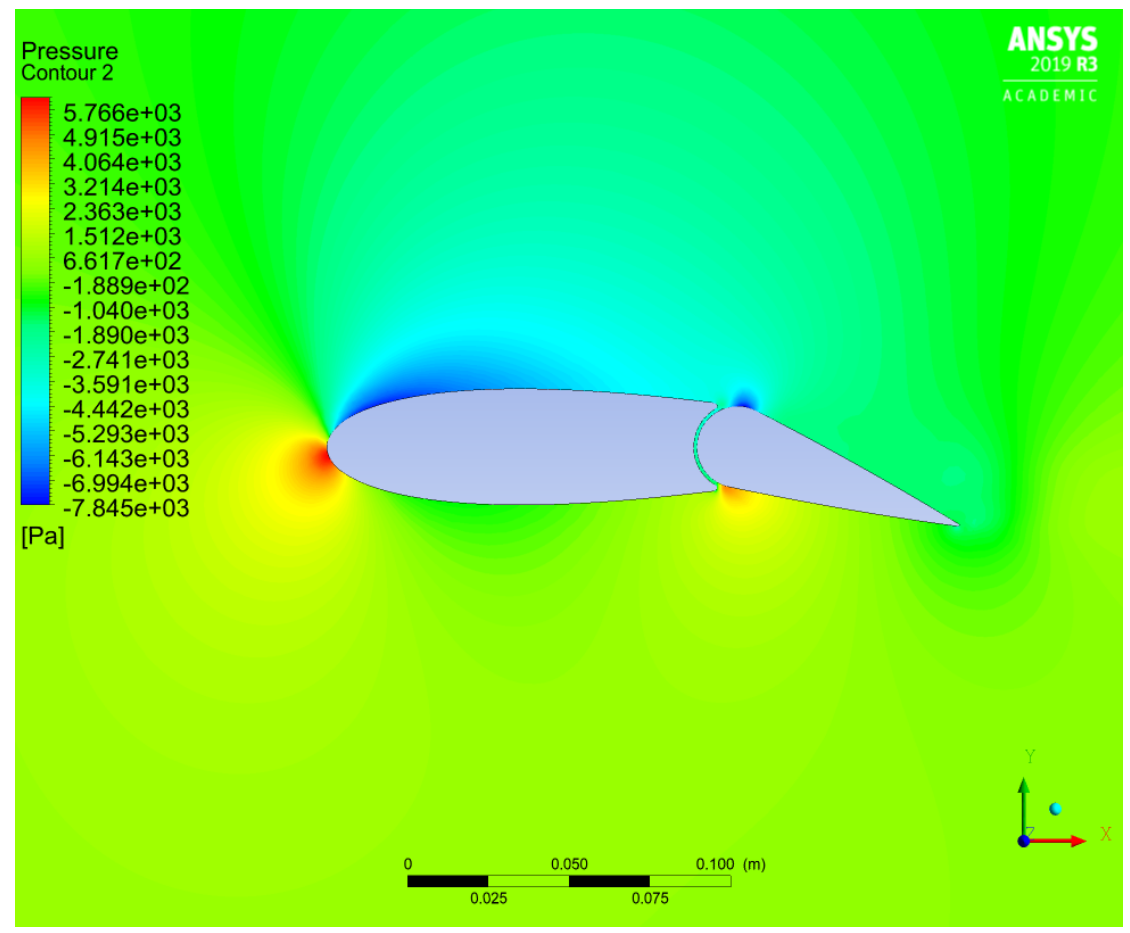

Figure 40: 20 deg Hinged Flap Pressure Contour

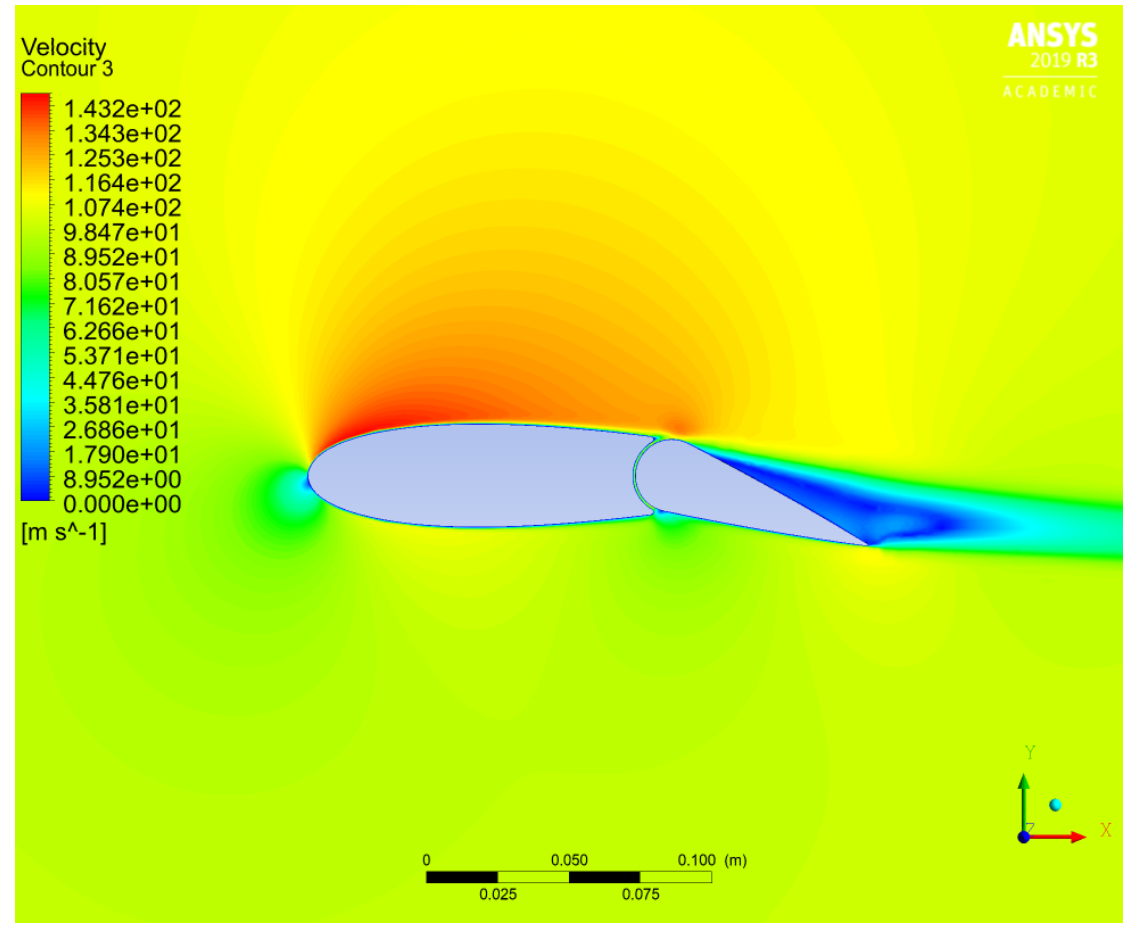

Figure 41: 20 deg Hinged Flap Velocity Contour 


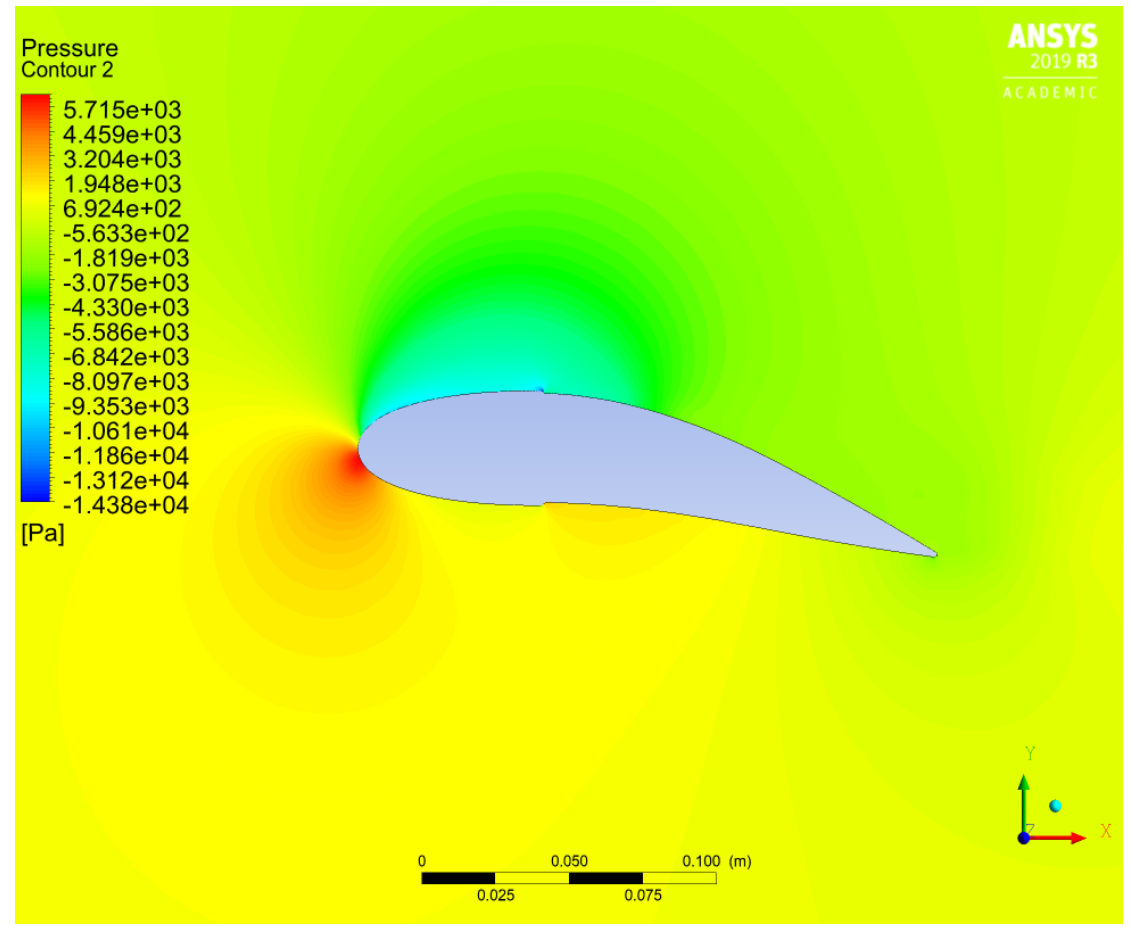

Figure 42: 20 deg Morphed Flap Pressure Contour

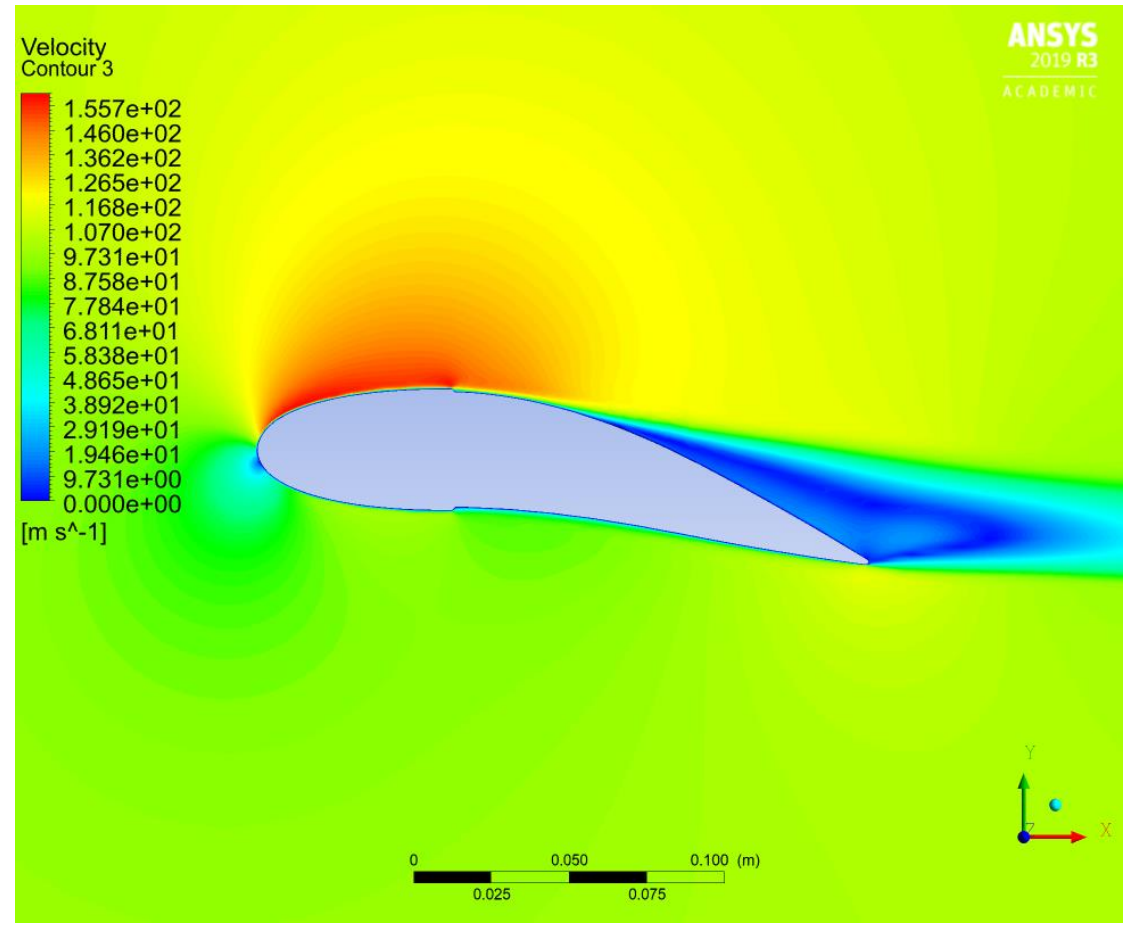

Figure 43: 20 deg Morphed Flap Velocity Contour 


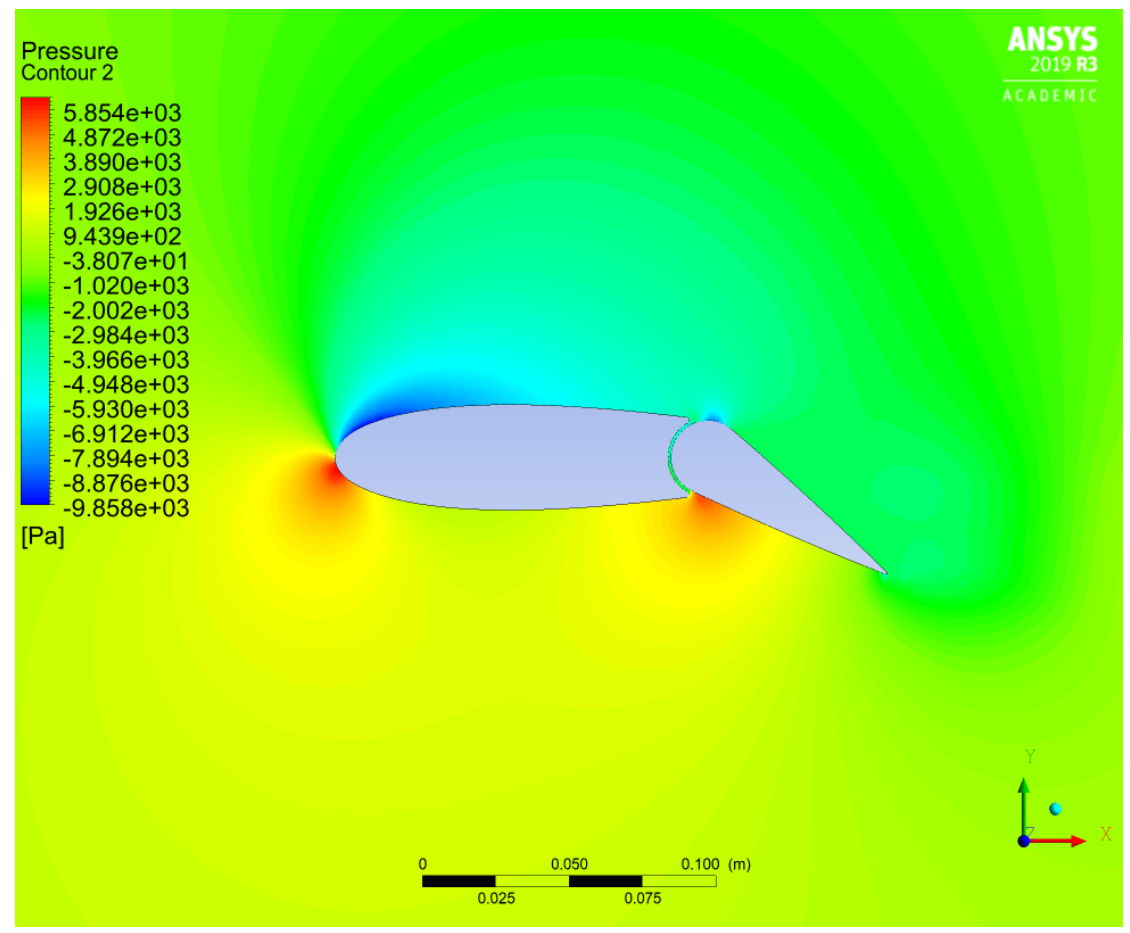

Figure 44: 30 deg Hinged Flap Pressure Contour

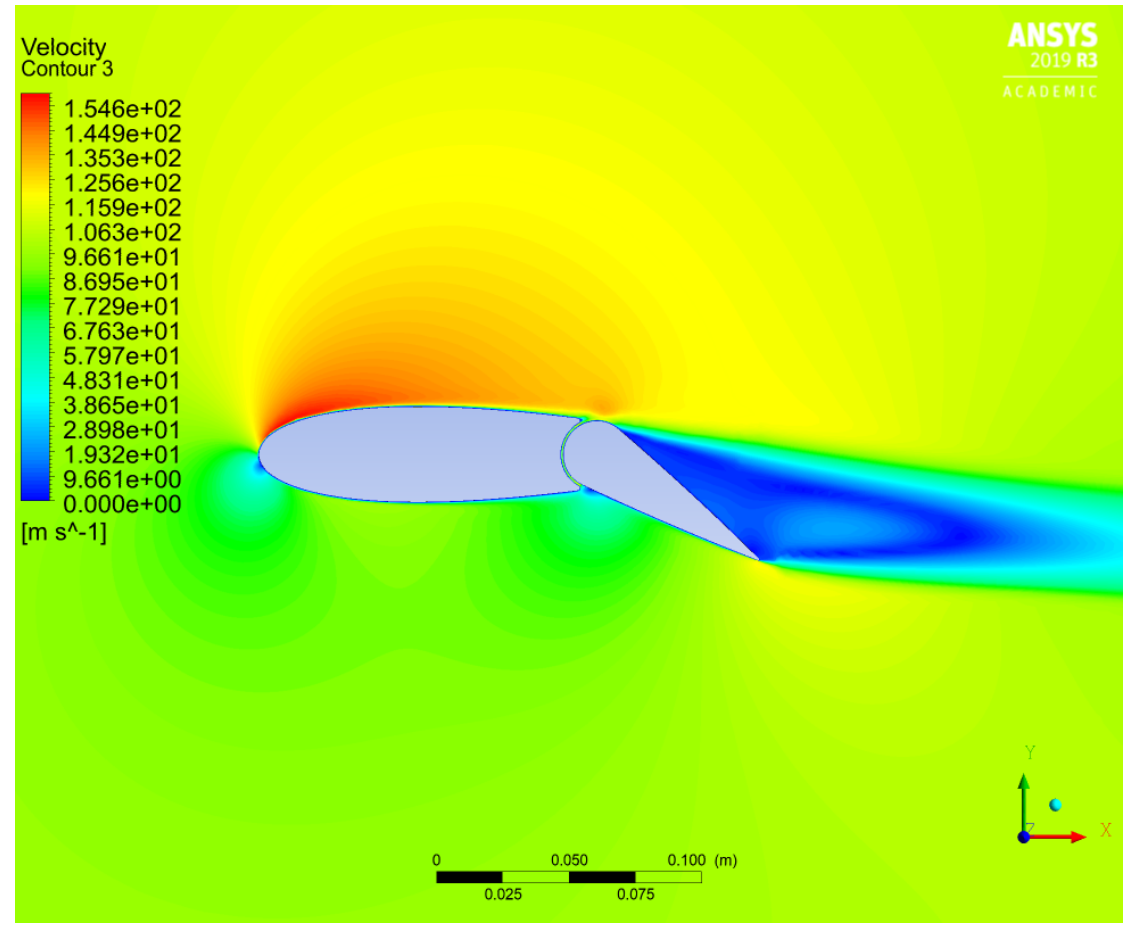

Figure 45: 30 deg Hinged Flap Velocity Contour 


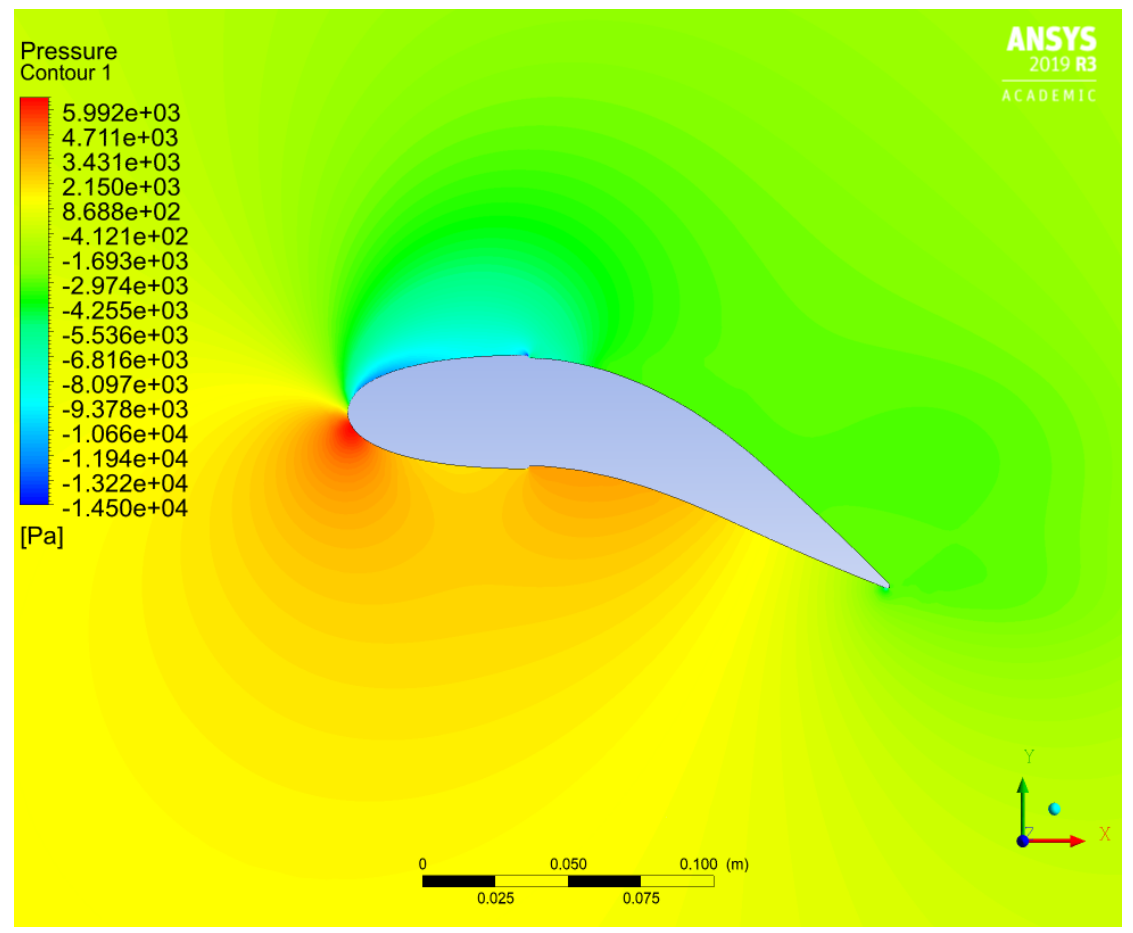

Figure 46: 30 deg Morphed Flap Pressure Contour

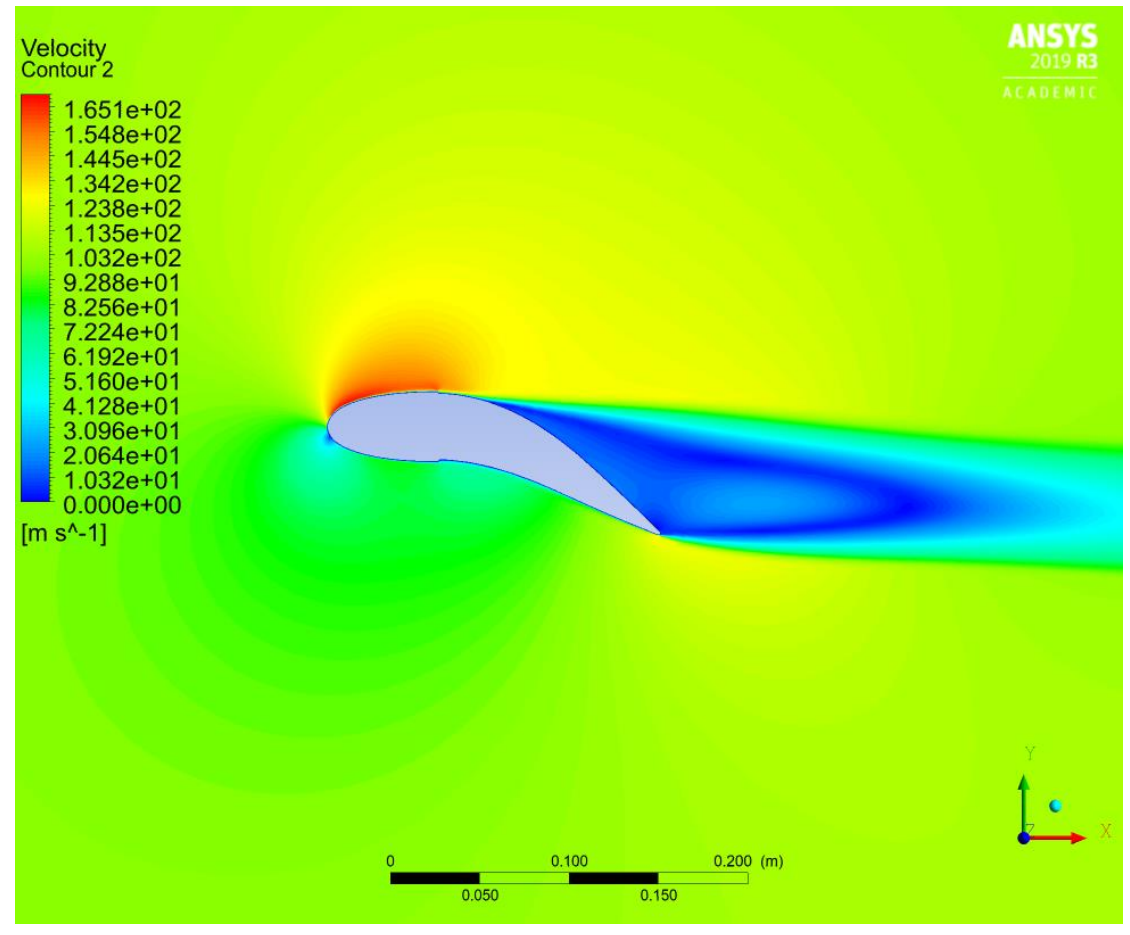

Figure 47: 30 deg Morphed Flap Velocity Contour 
Appendix C: Technical Drawings 


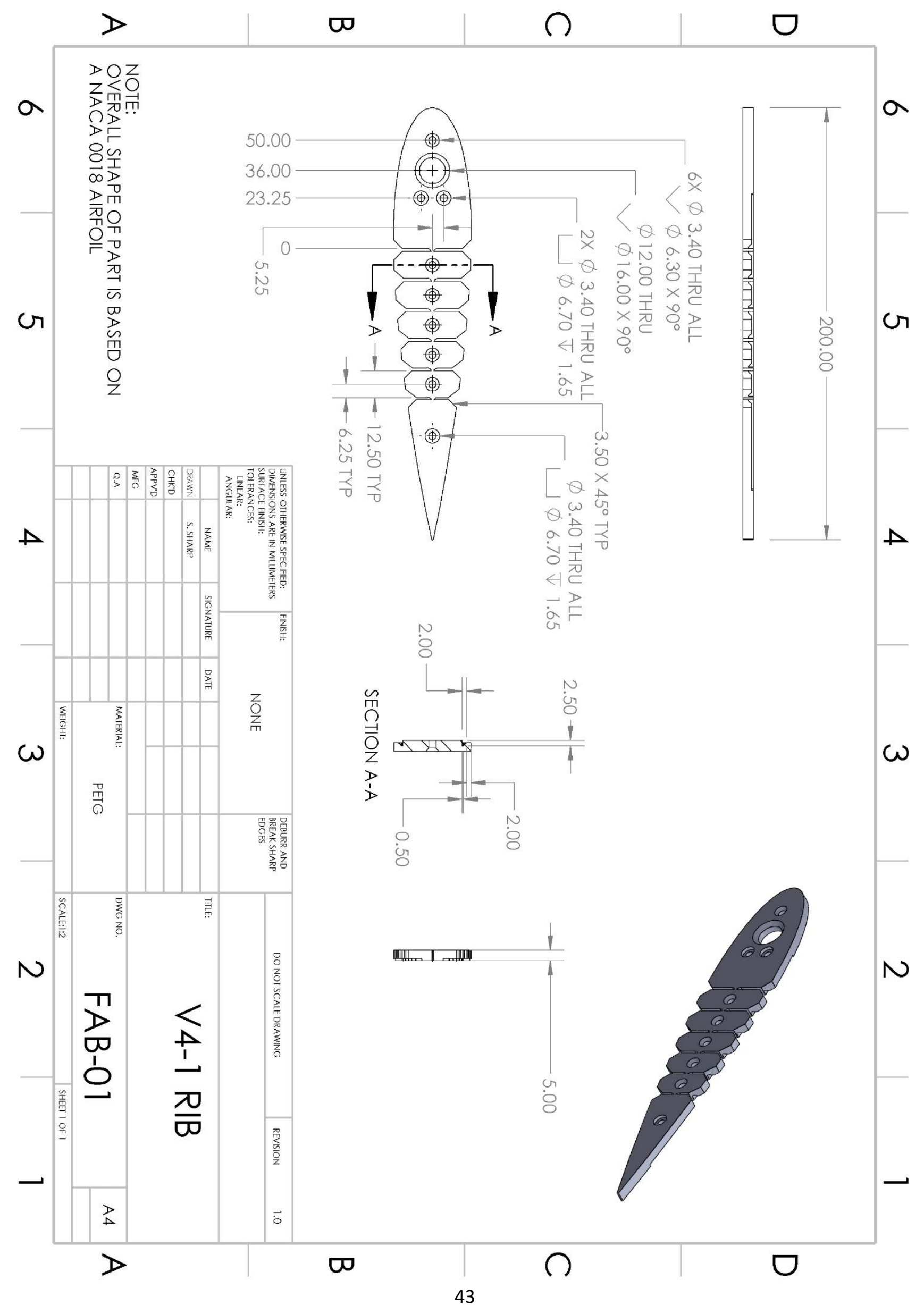




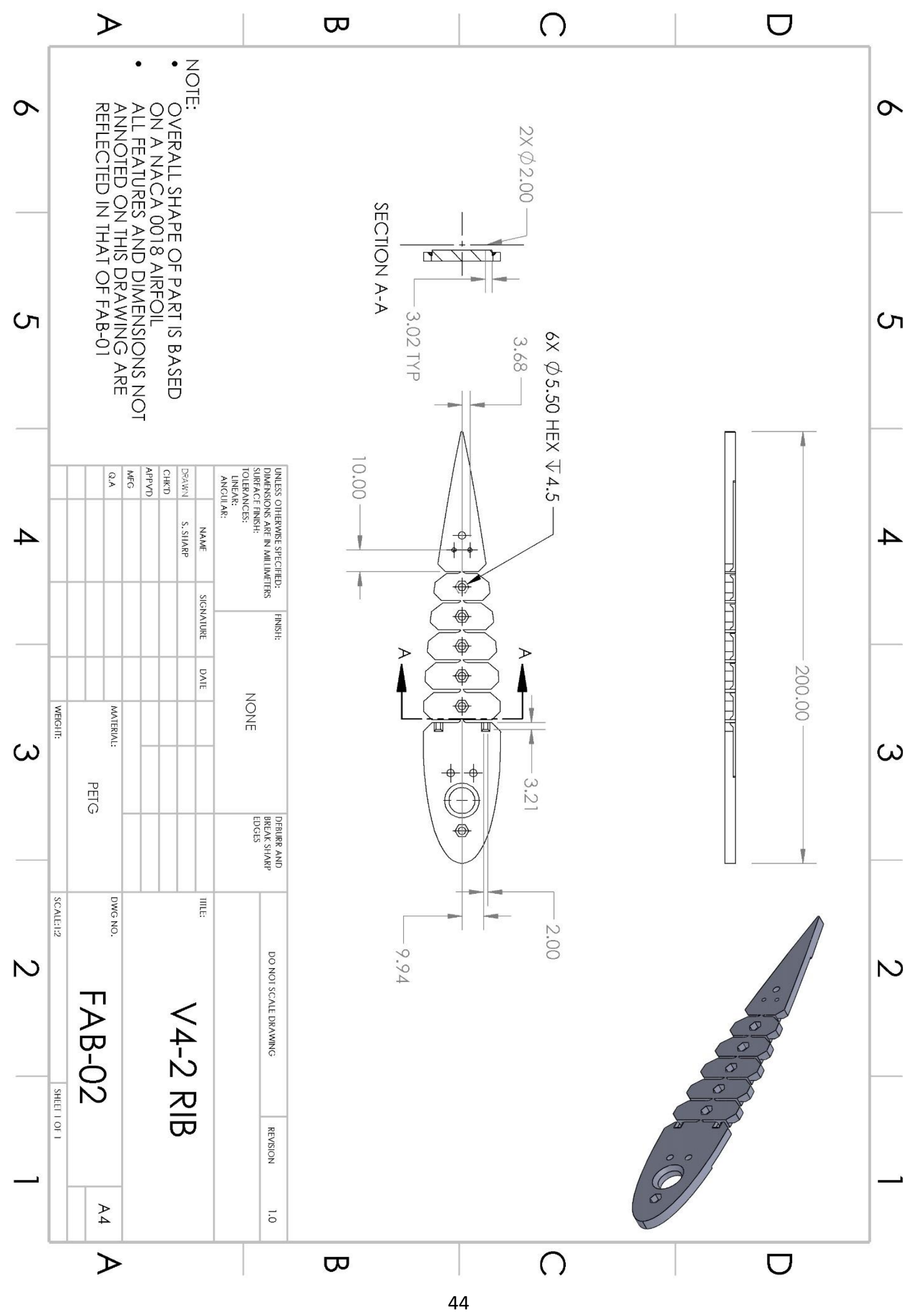




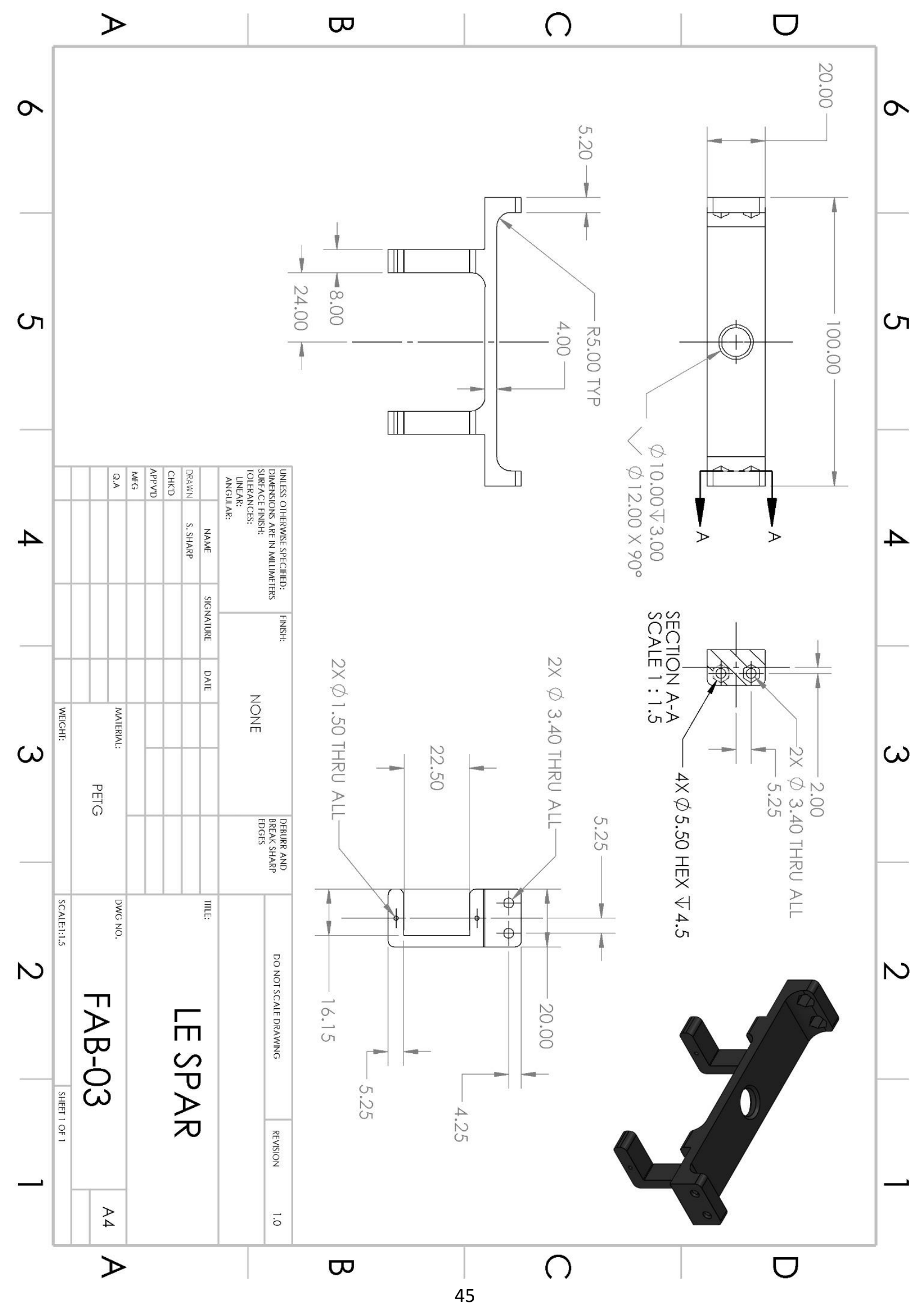




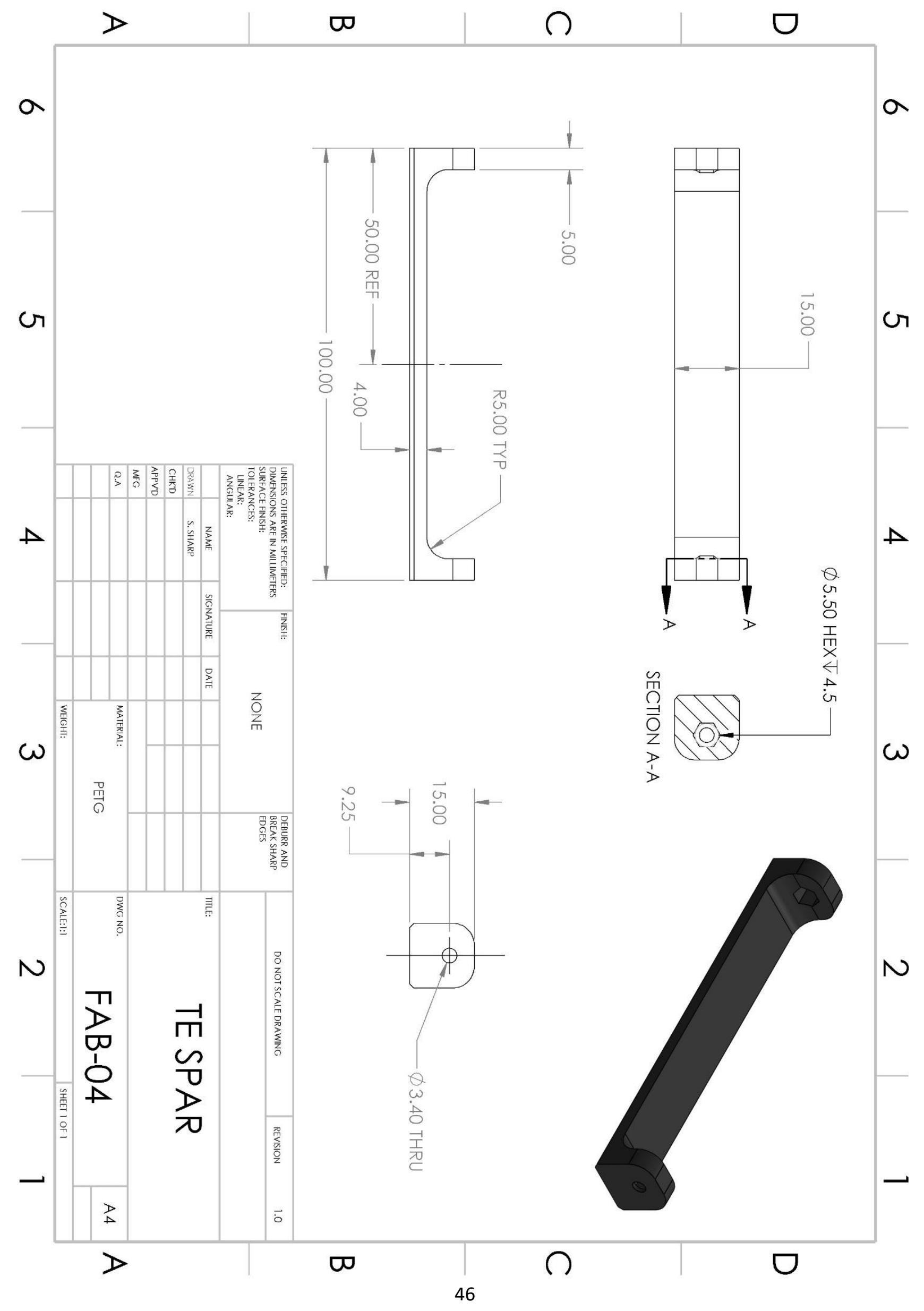




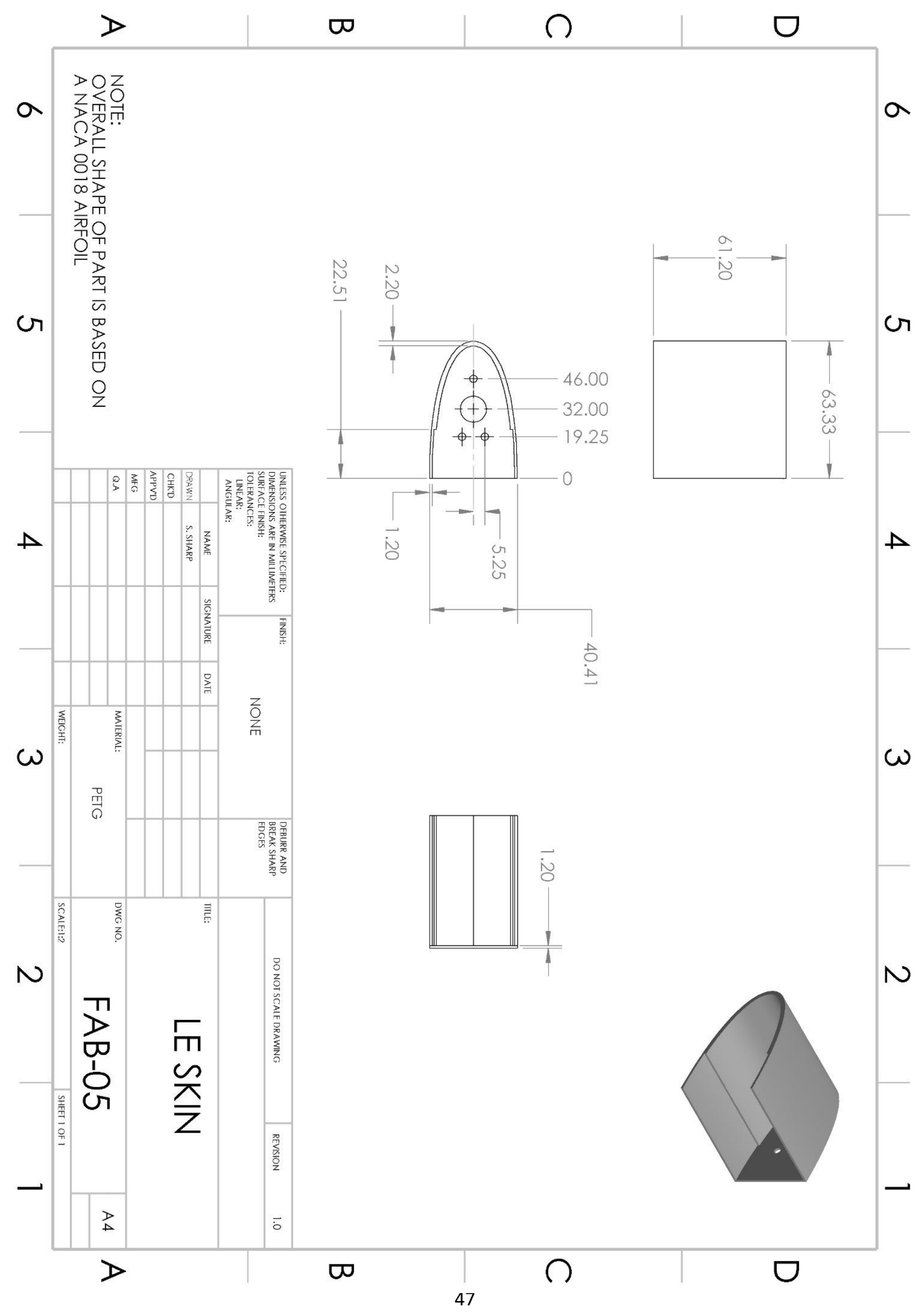




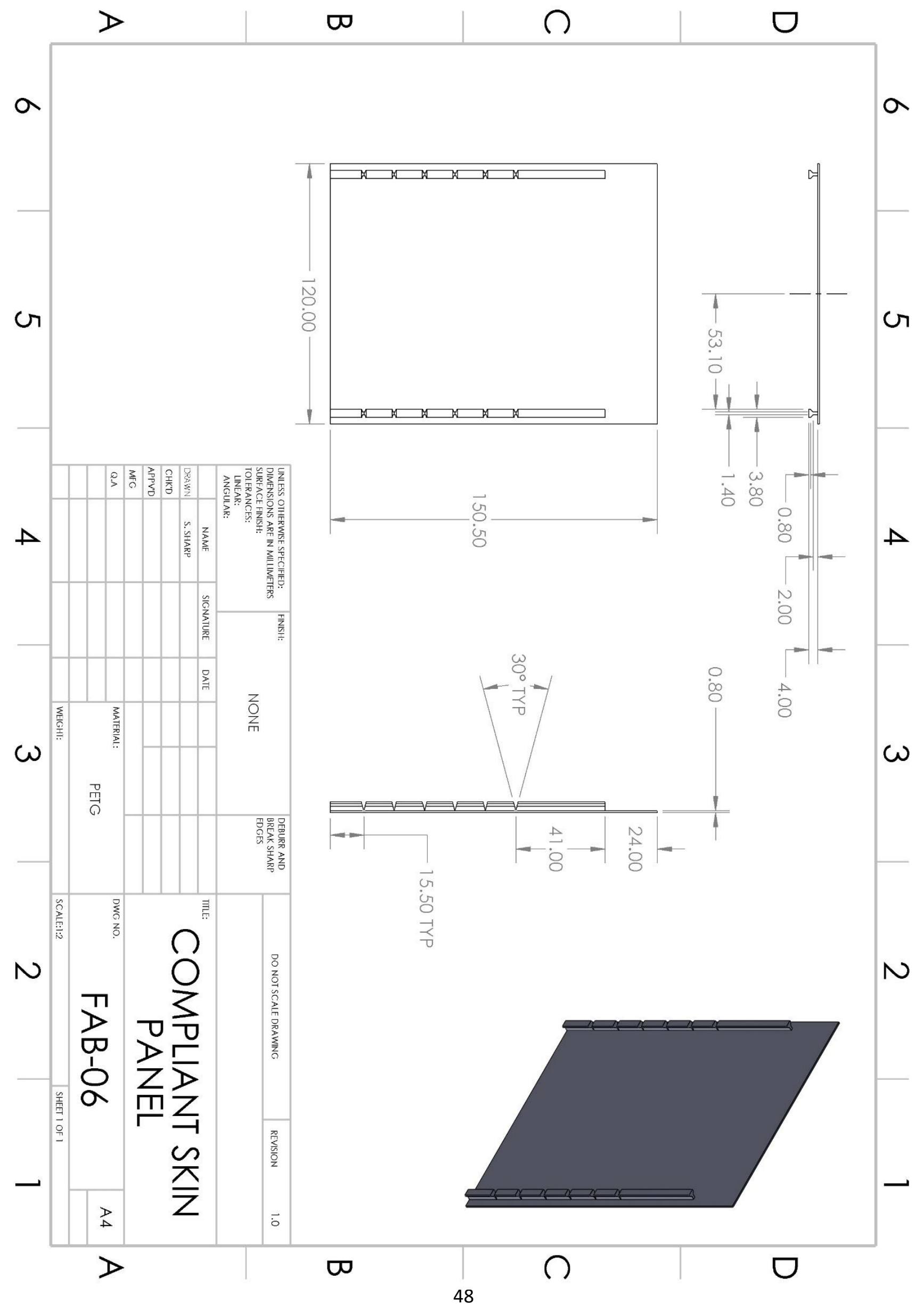

